\title{
La dinámica del desempleo en el Uruguay a través de la teoría de la reacción en cadena ${ }^{1}$
}

\author{
Martín Leites y Sylvina Porras
}

\section{Resumen}

En este artículo se analiza la dinámica del desempleo en el Uruguay en el marco de la teoría de la reacción en cadena y se aporta evidencia que explica la notable reducción del desempleo en los últimos años. Se confirma la incidencia de variables exógenas sobre la trayectoria del desempleo en el largo plazo y se descarta que su nivel de largo plazo gravite alrededor de un valor de equilibrio. Se constata la presencia de procesos de inercia en la demanda y oferta laboral y en los salarios, que interactúan entre sí y redundan en que los choques tengan efectos persistentes sobre el desempleo. Además, existen efectos de derrame complementarios que afectan la magnitud y duración de los efectos de los choques. Finalmente, se destaca que el crecimiento del acervo de capital y de su productividad explica parte de la significativa caída del desempleo en el Uruguay desde 2003.

Palabras clave

Desempleo, mercado de trabajo, oferta de mano de obra, salarios, medición, Uruguay Clasificación JEL J21, J23, J64

Autores

Martín Leites es Investigador del Instituto de Economía de la Universidad de la República, Uruguay.mleites@iecon.ccee.edu.uy

Sylvina Porras es Investigadora del Instituto de Economía de la Universidad de la República, Uruguay. sylvina@iecon.ccee.edu.uy

Los autores agradecen a Verónica Amarante por la revisión y los valiosos comentarios realizados sobre una versión previa de este documento. 


\section{Introducción}

La tasa de desempleo en el Uruguay se redujo 12 puntos porcentuales entre 2002 y 2011, y en los últimos años ha mantenido niveles históricamente bajos en comparación con su desempeño a través del tiempo. Esto sugiere la presencia de cierta inercia en su dinámica, cuestiona la existencia de una tasa natural de desempleo y abre interrogantes sobre cuáles son los factores que explican dicho proceso. En particular, cobra relevancia la indagación sobre cómo se vincula la evolución del desempleo de largo plazo con la acumulación de capital, variable que en ese período cobró un importante dinamismo, incrementando la capacidad productiva del país. En el gráfico 1 se observa que desde finales de la década de 1990, la dinámica de la formación bruta de capital fijo tuvo un comportamiento simétrico respecto a la evolución de la tasa de desempleo. Esto sugiere la hipótesis de que esta variable exógena al mercado de trabajo podría explicar parte de la caída de la tasa de desempleo a través de su impacto positivo sobre la demanda de trabajo.

\section{Gráfico 1}

Evolución de la formación bruta de capital fijo y de la tasa de desempleo, 1997-2011

(En miles de millones de pesos a precios de 2005 y porcentajes)

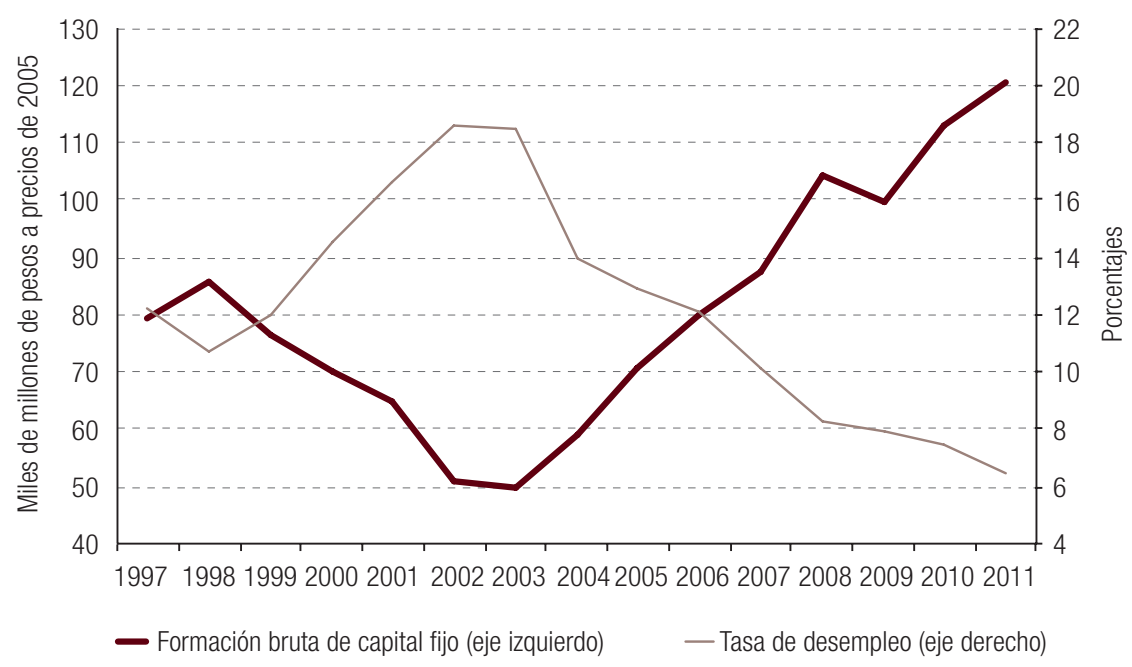

Fuente: Elaboración propia, sobre la base de datos del Instituto Nacional de Estadística (INE) y del Banco Central del Uruguay (BCU).

En los trabajos anteriores en que se estudia la dinámica del desempleo en el Uruguay se utilizan los enfoques más extendidos en la literatura económica: el de la tasa natural de desempleo y la hipótesis de histéresis. Sus resultados no son concluyentes respecto a la existencia de una tasa natural de desempleo y, si bien se confirma cierto comportamiento inercial, no se aportan elementos sobre los factores determinantes de su evolución. Los enfoques utilizados en estos trabajos presentan tres limitaciones fundamentales: i) operan con modelos uniecuacionales; ii) no permiten articular el comportamiento de corto y de largo plazo, y iii) descartan la incorporación de variables con tendencia para explicar el desempleo.

Esta investigación tiene como objetivo explicar la dinámica del desempleo en el Uruguay durante el período comprendido entre 1985 y 2011, aplicando el enfoque de la teoría de la reacción en 
cadena, que presenta algunas ventajas respecto a los abordajes más extendidos para responder las interrogantes planteadas. Este enfoque es más general y, en lugar de presuponer la convergencia del desempleo a un nivel de equilibrio o la permanencia de los efectos de los choques (histéresis), sugiere un modelo para evaluar empíricamente ambas hipótesis. En segundo lugar, es menos restrictivo y permite identificar los efectos sobre la tasa de desempleo de choques provenientes de variables exógenas al mercado de trabajo. De acuerdo con Bande y Karanassou (2009), uno de los aportes esenciales de la teoría de la reacción en cadena es que permite evaluar empíricamente la incidencia de las variaciones del acervo de capital sobre la tasa de desempleo, tanto en el corto como en el largo plazo. Este aspecto contrasta con los enfoques dominantes, que enfatizan que los desplazamientos de la trayectoria del capital no tienen ningún efecto de largo plazo sobre la tasa de desempleo (Layard, Nickell y Jackman, 1991).

En tercer lugar, ofrece una perspectiva integral que explica la evolución del desempleo de corto plazo de forma articulada con su trayectoria de largo plazo. Esto permite identificar los mecanismos de ajuste que operan en el mercado laboral, evaluar la persistencia de los efectos de un choque transitorio y analizar cómo variables exógenas al mercado de trabajo, y su interacción con los procesos de ajuste en la oferta y la demanda (efectos de derrame), pueden tener efectos persistentes o permanentes en el desempleo de largo plazo.

Hasta donde es de conocimiento de los autores, este enfoque no ha sido utilizado para estudiar el desempleo en países en desarrollo. Su aplicación resulta de interés, considerando algunas características de los mercados laborales de los países de la región, como la informalidad y el nivel de segmentación, así como su notorio dinamismo reciente (Weller, 2014; Banco Mundial, 2012). En este marco, el Uruguay no es la excepción y ha presentado en el período reciente niveles de desempleo históricamente bajos en relación con el contexto nacional y regional, lo que fundamenta la importancia de responder cómo la dinámica del desempleo fue afectada por los siguientes elementos: i) la presencia de factores de inercia; ii) variables exógenas al mercado de trabajo; iii) la existencia de efectos de derrame, y iv) la complementariedad de los procesos de ajuste. Contar con evidencia sobre estos aspectos contribuye a una mejor comprensión de la dinámica del desempleo y permite identificar cuáles fueron los principales determinantes de su caída reciente. En particular, esta investigación aporta evidencia sobre la incidencia de la acumulación del capital y de la productividad del trabajo sobre la caída del desempleo, variables que Weller (2014) sugiere que podrían expresar la relevancia del contexto productivo para explicar el dinamismo del empleo. Además, de forma indirecta, captura cómo el contexto productivo interactúa con los aspectos institucionales, generando mecanismos de propagación de los choques.

Los resultados obtenidos permiten descartar que la tasa de desempleo del Uruguay gravite en torno a un nivel de equilibrio y concluir que variables exógenas al mercado de trabajo, como la acumulación del capital, la productividad del capital o la población en edad de trabajar, inciden a través de los procesos de ajuste, generando efectos persistentes. Además, se confirma la existencia de complementariedad de los procesos de ajuste rezagados, lo que implica que, si se produjeran de forma aislada, más del 90\% del impacto inicial desaparecería al cuarto trimestre, mientras que si operan de forma simultánea esa situación se alcanza recién al décimo trimestre.

El artículo está organizado en cuatro secciones, además de esta Introducción. En primer lugar, se repasan las contribuciones de la teoría de la reacción en cadena y se presentan resultados de su aplicación en otros países. A continuación, se describe la metodología aplicada en esta investigación y luego se exponen los resultados. Finalmente, se resumen las conclusiones del trabajo. 


\section{Revisión de la literatura}

\section{Teoría de la reacción en cadena: concepto y aplicaciones}

Dentro de la corriente principal de la disciplina económica, la teoría sobre la tasa natural de desempleo, o en su versión más moderna la tasa de desempleo no aceleradora de la inflación (TDNAI), ha sido una de las más influyentes en los estudios sobre el desempleo a nivel macroeconómico. Apoyándose en la evidencia sobre el desempeño del mercado de trabajo en los países desarrollados durante las últimas tres décadas del siglo XX, Layard, Nickell y Jackman (1991) plantean la existencia del equilibrio sin pleno empleo y lo identifican como aquel nivel de desempleo que no acelera la inflación. La teoría de la reacción en cadena surge como un enfoque alternativo para el estudio de la tasa de desempleo, superando algunas de las limitaciones de la teoría de la TDNAI: permite modelizar la trayectoria del desempleo de forma más integral, al articular su comportamiento de corto y de largo plazo, evaluar la incidencia de variables exógenas al mercado de trabajo y explicar cuáles son los factores que determinan la persistencia o permanencia de choques transitorios ${ }^{2}$.

El enfoque de la teoría de la reacción en cadena se basa en un modelo multiecuacional del mercado de trabajo, en que los cambios en la tasa de desempleo son vistos como "reacciones en cadena" a las perturbaciones temporales y permanentes del mercado de trabajo.

Dichas reacciones del desempleo se producen a través de una red de interacción de procesos de ajuste rezagados de las variables relevantes del mercado de trabajo: oferta, demanda y salarios. El comportamiento de estas variables presenta cierta inercia o memoria, de modo que los ajustes no son inmediatos, lo que implica que sus valores presentes dependen de sus valores pasados y genera una fuente de persistencia de los efectos de los choques sobre la tasa de desempleo. La inercia de los procesos está bien documentada en la literatura sobre la teoría de la reacción en cadena (Karanassou, Sala y Salvador, 2008) y se debe a la presencia de costos de rotación laboral (de contratación, formación y despido) y escalonamiento de precios y salarios, y la existencia de mercados internos, efectos de la duración del desempleo y ajustes en la oferta de trabajo (por ejemplo, mediante la emigración). Otro elemento que desempeña un rol relevante es el hecho de que los procesos están interconectados entre sí y con variables exógenas, lo que redunda en que choques externos generen efectos de derrame, amplificando su efecto de corto plazo sobre la tasa de desempleo y extendiendo su duración, e impidiendo que la tasa de desempleo converja en el largo plazo a un nivel invariante (Karanassou y Snower, 1997 y 1998).

El abordaje empírico del estudio del desempleo bajo el enfoque de la teoría de la reacción en cadena consiste en la modelización de un sistema de ecuaciones como el que se presenta a continuación ${ }^{3}$ :

$$
\begin{gathered}
n_{t}=\alpha_{1} n_{t-1}+\beta_{1} k_{t}-\gamma_{1} w_{t} \\
l_{t}=\alpha_{2} l_{t-1}+\beta_{2} z_{t}+\gamma_{2} w_{t} \\
w_{t}=\beta_{3} x_{t}-\gamma_{3} u_{t}
\end{gathered}
$$

\footnotetext{
2 El enfoque de la teoría de la reacción en cadena fue desarrollado en Karanassou y Snower (1996). Véase una revisión, entre otros, en Karanassou (1998), Karanassou y Snower (1997, 1998 y 2000) y Henry, Karanassou y Snower (2000).

3 Los fundamentos teóricos que sustentan la especificación de cada ecuación son desarrollados en Karanassou y Snower (2000). Un resumen se presenta en Leites y Porras (2013).
} 
donde $n, l$ y $w$ son variables endógenas y representan la demanda de trabajo, la oferta de trabajo y el salario real, respectivamente ${ }^{4}$. Excepto la tasa de desempleo $(u)$, las demás variables del sistema están expresadas en logaritmos, por lo que dicha tasa se obtiene a partir de la siguiente expresión:

$$
u_{t} \cong l_{t}+n_{t}
$$

Los coeficientes $\alpha_{i}$ (con $0<\alpha_{i}<1$ ) expresan los efectos de la inercia de los procesos; los parámetros $\gamma_{i}$ las interacciones entre las variables endógenas (efectos de derrame) y $^{5} \beta_{i}$ las elasticidades de corto plazo de las variables endógenas respecto a los cambios en las variables exógenas, como son el acervo de capital $(k)$, la población en edad de trabajar $(z)$ y factores de presión sobre los precios $(x)$.

Esta modelización de la teoría de la reacción en cadena permite superar una de las principales limitaciones del enfoque de la TDNAI, en el que se asume que la modelización de la tasa de desempleo solo puede incluir variables exógenas sin tendencia (hipótesis de invarianza), lo que implica que las perturbaciones permanentes de variables exógenas conducen a cambios compensatorios en las curvas de demanda y oferta de trabajo, a fin de restablecer la tasa de desempleo a su nivel de equilibrio de largo plazo original (Layard, Nickell y Jackman, 1991). Una modelización menos restrictiva (hipótesis de invarianza débil) es aquella que incluye una combinación lineal estacionaria de variables con tendencia exógenas al mercado laboral (Phelps, 1994). Karanassou y Snower (2004) demuestran que esas restricciones de invarianza no se cumplen y que variables con tendencia como el acervo de capital, el cambio tecnológico, la productividad del trabajo o la población en edad de trabajar son variables relevantes para explicar el comportamiento del mercado laboral y, en consecuencia, el desempleo. El requerimiento es que cada variable endógena con tendencia $(l, n$ y $w)$ se equilibre en conjunto con las variables explicativas.

Este enfoque se ha aplicado al estudio de la tasa de desempleo del Reino Unido, los Estados Unidos, Dinamarca, Australia, Portugal, España y un conjunto de países de la Unión Europea (Karanassou y Snower, 1998; Henry, Karanassou y Snower, 2000; Karanassou, Sala y Snower, 2003; Bande, 2002; Bande y Karanassou, 2009; Karanassou, Sala y Salvador, 2008; Karanassou y Sala, 2008 y 2010; González y Sala, 2011) ${ }^{6}$. En estos estudios se resalta la importancia de este enfoque en comparación con un modelo uniecuacional de la tasa de desempleo, y se concluye que las decisiones de política económica no deben basarse en lo que determina la tasa natural de desempleo, dado que se encuentra que la tasa de desempleo no gravita en torno a este nivel de equilibrio.

En todos los casos se confirma la existencia de inercia en los procesos de ajuste y solo se identifican diferencias en la velocidad de los mismos. La demanda de trabajo se ajusta con mayor rapidez en países como Dinamarca y Australia ( $\alpha_{1}=0,2$ aproximadamente), y en el otro extremo se ubica la inercia estimada para la Unión Europea en su conjunto $(0,94)$, seguida por la de los Estados Unidos y el Reino Unido (0,7 aproximadamente). Se argumenta que el resultado correspondiente a Dinamarca refleja una mayor flexibilidad de su mercado de trabajo respecto a los demás países de la Organización para la Cooperación y el Desarrollo Económicos (OCDE), en cuanto a la legislación de protección al empleo (Karanassou, Sala y Salvador, 2008). En el caso de la oferta de trabajo, las ecuaciones presentan coeficientes de inercia más homogéneos y en niveles relativamente elevados

\footnotetext{
4 Para simplificar, en la ecuación de salario se excluye la incidencia del salario rezagado, siguiendo el ejemplo de Karanassou, Sala y Snower (2009). Con el mismo objetivo se excluyeron los términos de error y constantes.

5 En esta versión sencilla, el salario incide en la demanda y en la oferta de trabajo, mientras que el desempleo afecta también la determinación del nivel de salario. Mayores niveles de desocupación disminuirían el poder de negociación relativo de los trabajadores empleados, haciendo creíble la amenaza de despido, lo que ejercería una presión a la baja de los salarios.

6 En el estudio sobre la Unión Europea se incluye a los siguientes países: Alemania, Austria, Bélgica, Dinamarca, España, Finlandia, Francia, Italia, Países Bajos, Reino Unido y Suecia.
} 
$\left(\alpha_{2} \text { entre } 0,6 \text { y } 0,92\right)^{7}$, lo que indica la existencia de costos de entrada y salida de la fuerza de trabajo. En todos los casos, la ecuación de salarios incluye también rezagos de la variable dependiente. Dejando de lado los datos de Dinamarca y Australia, los coeficientes de inercia de los salarios son también relativamente homogéneos y oscilan entre 0,55 y 0,83. Al igual que en el caso de la demanda de trabajo, esos dos países son los que presentan los procesos de ajuste más rápidos $(0,32$ y 0,24 , respectivamente).

Por otro lado, Karanassou y Snower (1998) encuentran que más de la mitad de los cambios de la tasa de desempleo del Reino Unido entre 1980 y 1995 se debieron a la contribución de los ajustes rezagados de las variables del mercado de trabajo en el mediano plazo. Además, indican que los procesos de ajuste han sido complementarios, lo que genera efectos más significativos sobre la tasa de desempleo. Henry, Karanassou y Snower (2000) estiman, en el caso del Reino Unido, que un choque temporal positivo de la demanda de trabajo ${ }^{8}$ provoca una reducción gradual de la tasa de desempleo, de modo que el 90\% del efecto inicial recién desaparece después de cuatro años. Por su parte, un choque temporal sobre el salario real produce un fuerte incremento inicial de la tasa de desempleo, que luego inicia un proceso gradual de caída, logrando completar el $90 \%$ del ajuste total recién a los 12 años, mientras que el 90\% del ajuste ante un choque proveniente de la oferta de trabajo desaparece a los diez años.

En las ecuaciones de demanda laboral de todos los países se incluyó como variable explicativa el salario real. La elasticidad del empleo de largo plazo respecto a los cambios en los salarios toma el signo esperado para todos los países, cumpliendo la ley de la demanda ${ }^{9}$, pero los valores difieren en magnitud. Dicha elasticidad se ubica por encima de 0,6 como valor absoluto en una de las estimaciones para España, así como en los casos de los Estados Unidos y Dinamarca (en los que toma valores entre 1 y 2). El resto de las estimaciones indican una elasticidad de largo plazo de la demanda laboral respecto al precio menor que 0,5.

Además de las variables mencionadas, sus rezagos e interacciones, las ecuaciones incluyen otras variables exógenas a los mercados de trabajo como variables explicativas. Una de las variables más utilizadas es el acervo de capital, que se incluye en las ecuaciones de demanda laboral. Las estimaciones indican una elasticidad de largo plazo positiva que oscila entre 0,3 y 1, lo que implica un efecto de largo plazo también sobre el desempleo. En el estudio referente a Australia, Karanassou y Sala (2010) concluyen que en la década de 1990 y principios de la década de 2000, la acumulación de capital fue el factor que tuvo mayor incidencia en la trayectoria del desempleo de dicho país. Sobre la base de estos resultados, los autores cuestionan el enfoque de la TDNAl y descartan que la trayectoria del desempleo solo responda a choques temporales o a cambios en las instituciones laborales. Karanassou y Sala (2008) encuentran evidencia en el mismo sentido para el caso de España entre los años 1970 y 2005. Aplican el enfoque de la teoría de la reacción en cadena para evaluar la influencia de las variaciones de las contribuciones a la seguridad social, los impuestos indirectos, la riqueza financiera y la acumulación de capital sobre la evolución de la tasa de desempleo de España. Encuentran que la acumulación de capital es el factor que en mayor medida explica dicha evolución.

\section{Estudios sobre el desempleo en el Uruguay}

En algunos estudios anteriores se ha abordado el tema del desempleo en el Uruguay utilizando como marco los enfoques más extendidos: el de la tasa natural de desempleo y la hipótesis de histéresis.

\footnotetext{
7 Para el Reino Unido hay dos estimaciones diferentes, una con un coeficiente de inercia de 0,45 y la otra con uno de 0,75.

8 Toman como supuesto un choque que implica una variación inicial de 1 punto porcentual de la tasa de desempleo.

9 En el trabajo de Karanassou y Snower (1998) referente al Reino Unido, esta elasticidad se presenta con signo positivo, pero en el documento no se realiza ningún comentario al respecto.
} 
En estos trabajos se encuentra, por un lado, que no existe evidencia empírica concluyente respecto a la existencia de una tasa natural de desempleo a la que converja la economía del Uruguay y, en otros casos, no se rechaza la presencia de raíz unitaria en la serie de desempleo del país o, al menos, no se descarta que perturbaciones de naturaleza transitoria tengan efectos persistentes sobre el desempleo.

Efectivamente, Borraz y Tubio (2009) rechazan la existencia de la TDNAl en el caso uruguayo en el período comprendido entre 1978 y 2009 y, si bien encuentran una relación negativa entre la inflación y el desempleo, el método utilizado (curva de Phillips aumentada por expectativas) no les permitió inferir causalidad ${ }^{10}$. Sin embargo, utilizando otra metodología (filtro de Kalman, modelización univariada), estiman una tasa "natural" de desempleo independiente de la inflación del 10,64\% para 2009, por lo que el nivel que la tasa registró efectivamente entre 2009 y 2011 se ubicaría muy por debajo de su "nivel natural".

Rodríguez (1998), por su parte, encuentra evidencia favorable a la hipótesis de histéresis al analizar la evolución de la tasa de desempleo de Montevideo en el período comprendido entre 1984 y 1996. En el mismo sentido apuntan los resultados obtenidos por Badagián y otros (2001), quienes estudian la dinámica de la tasa trimestral de desempleo de la capital del país en el período comprendido entre el cuarto trimestre de 1983 y el segundo trimestre de 2001 y concluyen que el desempleo se comporta como un proceso estocástico con memoria larga (incluso suponiendo quiebres estructurales), lo que sería inconsistente con la existencia de una tasa natural de desempleo estacionaria. En otro estudio, también sobre la tasa de desempleo de Montevideo, en este caso del período 1968-1997, se concluye que los efectos de las perturbaciones de naturaleza transitoria tienden a desaparecer, pero registran una alta persistencia (Spremolla, 2001).

Esta evidencia implica la existencia de cierto comportamiento inercial de la tasa de desempleo, que podría explicarse por procesos con memoria en las variables relevantes del mercado de trabajo, como la demanda, la oferta y la formación de salarios. Esto supondría la existencia de ciertas rigideces en el mercado laboral y la no existencia de ajustes automáticos.

La evidencia disponible hasta ahora, en general, no es concluyente. El dinamismo reciente del mercado de trabajo del Uruguay abre la interrogante sobre qué factores podrían estar determinando los bajos niveles de desempleo registrados en los últimos años. Weller (2014) plantea que la evolución reciente del desempleo en los países de América Latina y el Caribe estaría explicada por un contexto productivo favorable (crecimiento económico, mejora de la productividad y comportamiento de otros factores productivos), así como por aspectos institucionales. El abordaje de este tema a través de la teoría de la reacción en cadena permitirá aportar evidencia directa sobre la relevancia de la productividad de los factores y de la acumulación del capital para explicar el desempleo. Asimismo, proporcionará evidencia indirecta sobre cómo los factores institucionales y del contexto productivo interactúan para amplificar los efectos de los choques.

\section{Metodología}

Se estima un modelo dinámico multiecuacional para explicar la evolución del desempleo en el Uruguay, teniendo como referencia el enfoque de la teoría de la reacción en cadena. Karanassou y Snower (2000) desarrollaron los fundamentos teóricos subyacentes de la modelización de cada una de las ecuaciones del sistema.

La ecuación de la demanda de trabajo se configura como una demanda derivada de la de bienes y servicios finales, a partir de la maximización del beneficio de las empresas que operan en

\footnotetext{
${ }^{10}$ A juicio de los autores, tanto la inflación como el desempleo se definen por procesos determinados por variables estructurales independientes.
} 
mercados no competitivos y en presencia de costos de ajuste. En este marco, la demanda de trabajo queda dependiendo, en primer lugar, de su propio pasado (variable dependiente rezagada), lo que indica la existencia de inercia, debido a los costos de ajuste que enfrentan los empleadores a la hora de contratar o despedir trabajadores. Para que la demanda de trabajo sea dinámicamente estable, el coeficiente de inercia debe ser inferior a la unidad. Además, el valor de dicho coeficiente da cuenta de la velocidad de ajuste de la demanda de trabajo ante choques externos: cuanto más cercano a la unidad sea el coeficiente, más lento será el ajuste y cuanto más cercano a 0 sea el coeficiente, más rápido será el ajuste. La demanda de trabajo también depende de su propio precio (salario real), del acervo de capital y de la productividad del capital.

En la ecuación de la oferta de trabajo se incluye al menos un rezago de la variable dependiente, aceptando el supuesto de que existen costos de entrada y de salida de la fuerza de trabajo. Esto habilita la presencia de inercia en las decisiones de la oferta laboral. Además, su variación depende también de otros factores: i) el crecimiento de la población en edad de trabajar; ii) el salario real, sin un signo definido a priori, dado que la oferta puede reaccionar positivamente ante aumentos del salario (efecto sustitución) o negativamente (efecto renta), y iii) la tasa de desempleo, cuyo efecto podría ser negativo en la medida en que opere el efecto del trabajador desalentado (que consiste en que una parte de la fuerza laboral abandona la búsqueda de trabajo, frente a las escasas oportunidades existentes) o positivo si predomina el efecto del trabajador añadido (que consiste en que, ante aumentos de la cantidad de desocupados en el hogar, más personas ingresen al mercado, intentando compensar la reducción de los ingresos).

Finalmente, la ecuación de salarios contiene al menos un rezago de la variable dependiente, asociado a la inercia explicada por el efecto de escalonamiento de salarios. Este se deriva del proceso de negociación entre trabajadores y empresas, que acuerdan incrementos salariales considerando la trayectoria pasada, por lo que la influencia de los rezagos es inmediata. De acuerdo con Bande (2002), a nivel teórico, el modelo descriptivo del comportamiento del salario agregado se basa en los procesos de escalonamiento salarial descritos por Taylor (1979). Se acepta el supuesto de que los contratos tienen una vigencia de un período, pero se determinan en dos momentos del tiempo, la mitad a principios de año y la otra mitad a mediados del período. Ello se traduce en que el salario corriente dependa del salario del período anterior ${ }^{11}$. Además, los salarios dependen de la capacidad de negociación salarial, que se puede modelizar como una función del nivel de desempleo. La significación de esta variable estaría capturando el efecto del trabajador interno (insider membership effect) en la capacidad de negociación para la fijación de salarios; según este efecto, una parte de los participantes en el mercado de trabajo tienen una posición de privilegio en relación con los trabajadores externos, explicada básicamente por los costos de rotación de personal que enfrentan las firmas ${ }^{12}$. Mayores niveles de desocupación disminuirían el poder de negociación de los trabajadores internos, lo que ejercería una presión a la baja de los salarios, situación contraria a la que ocurre cuando el desempleo es bajo. La teoría más tradicional también incluye como factor explicativo de las variaciones salariales los cambios en la productividad del trabajo ${ }^{13}$.

A partir de los fundamentos expuestos, se estima el siguiente sistema:

$$
\begin{gathered}
n_{t}=c_{n}+\alpha_{n} n_{t-1}+\gamma_{1} w_{t}+\beta_{1} k_{t}+\beta_{2} p r k_{t}+\varepsilon_{n} \\
l_{t}=c_{l}+\alpha_{l} l_{t-1}+\gamma_{2} w_{t}+\gamma_{3} u_{t}+\beta_{3} z_{t}+\varepsilon_{l}
\end{gathered}
$$

\footnotetext{
11 Véase el desarrollo en Bande (2002).

12 Montuenga y Ramos (2005) plantean distintos fundamentos para explicar la inclusión de la tasa de desempleo como determinante del salario.

${ }^{13}$ Los modelos de salario de eficiencia sugieren que la productividad del trabajo podría ser endógena al sistema, aspecto que hasta el momento no ha sido considerado en la teoría de la reacción en cadena.
} 


$$
w_{t}=c_{w}+\alpha_{w} w_{t-1}+\gamma_{4} u_{t}+\beta_{4} p r n_{t}+\varepsilon_{w}
$$

Siendo:

$$
u_{t}=l_{t}-n_{t}
$$

donde $n_{t}$ es el número de ocupados, $w_{t}$ el salario real medio, $k_{t}$ el acervo de capital, prk $k_{t}$ la productividad aparente del capital, $l_{t}$ la cantidad de personas activas en el mercado de trabajo, $u_{t}$ la tasa de desempleo, $z_{t}$ la población en edad de trabajar y $p r n_{t}$ la productividad aparente del trabajo. Todas las variables, excepto la tasa de desempleo, están expresadas en logaritmos, por lo que los coeficientes estimados correspondientes a cada variable explicativa representan la elasticidad de corto plazo de la variable dependiente respecto a variaciones de dichas variables. Además, $c_{i}$ y $\varepsilon_{i}$ (con $i=n, l$ y $w$ ) representan la constante y el término de error de cada ecuación, respectivamente.

Una vez estimado el sistema, se propone contrastar las siguientes hipótesis:

- Ha. Presencia del efecto inercia. Para responder sobre este aspecto, se analiza la significación de las variables endógenas rezagadas en cada ecuación. En términos del modelo especificado, la presencia del efecto inercia implica rechazar Ha: $\alpha_{n}=0 ; \alpha_{l}=0 ; \alpha_{w}=0$.

- $\quad \mathrm{Hb}$. Incidencia de variables exógenas al mercado de trabajo. Se evalúa la significación de las siguientes variables: acervo de capital, productividad del capital, población en edad de trabajar y productividad del trabajo. Si se cumple alguna o algunas de las siguientes hipótesis: $\beta_{1} \neq 0 ; \beta_{2} \neq 0 ; \beta_{3} \neq 0 ; \beta_{4} \neq 0$, significa que variables con tendencia exógenas al mercado de trabajo incidirán en el nivel de la tasa de desempleo.

- Hc. Presencia de efectos de derrame. En el marco del modelo especificado, ello se cumple si se confirma alguna o algunas de las siguientes hipótesis: $\gamma_{1} \neq 0 ; \gamma_{2} \neq 0 ; \gamma_{3} \neq 0 ; \gamma_{4} \neq 0$. Esto implicaría que existe interacción entre las ecuaciones y, en consecuencia, un choque que se procese sobre una de las variables endógenas generará efectos de derrame sobre las otras, lo que puede provocar que el proceso de ajuste del desempleo sea aún más prolongado.

La contrastación de las hipótesis anteriores surge de la evaluación de la significación estadística de los coeficientes estimados en cada ecuación. De acuerdo a la teoría de la reacción en cadena, el hecho de que no se rechacen las hipótesis Ha y Hc implica que choques temporales tendrán un efecto persistente sobre la tasa de desempleo. Para evaluar dicha persistencia se utilizaron técnicas de simulación a partir de las funciones de impulso y respuesta.

- Hd. Hipótesis de complementariedad en los procesos de ajuste rezagado. Otro de los aspectos señalados en la literatura sobre el tema es que los efectos sobre el desempleo pueden amplificarse y/o prolongarse en el tiempo en la medida en que exista complementariedad en los procesos de ajuste. Como una forma de contrastar dicha hipótesis en el caso del Uruguay, se sigue a Henry, Karanassou y Snower (2000), y mediante técnicas de simulación se comparan los efectos de un choque sobre el desempleo en las siguientes dos simulaciones: una en que se considera que operan todos los efectos de forma simultánea, y otra que consiste en sumar las influencias individuales de cada ecuación como si operaran de forma separada.

Finalmente, como objetivo secundario, se propone indagar en los determinantes de la reciente caída del desempleo en el Uruguay, explorando cuán importante ha sido la acumulación de capital en la dinámica del desempleo de los últimos años. Para ello, siguiendo a Karanassou y Snower (1998), se realiza un ejercicio de simulación para identificar los factores que han contribuido en mayor medida a la caída del desempleo entre 2003 y 2011.

Las ecuaciones fueron estimadas usando la modelización autorregresiva con rezagos distribuidos de orden $p$ y $q$, analizando la cointegración de las variables incluidas en la modelización 
a partir de la metodología propuesta en los trabajos de Pesaran y Shin (1995), Pesaran, Shin y Smith (1996 y 2001) y Pesaran (1997), con $p$ y $q$ correspondientes al orden de rezagos de las variables dependiente e independiente, respectivamente.

Esta metodología es la utilizada en la literatura anterior en que se aplica el enfoque de la teoría de la reacción en cadena, debido a que presenta ciertas ventajas respecto a las técnicas de cointegración habitualmente utilizadas para estimar las relaciones de largo plazo y los mecanismos de ajuste de corto plazo. En primer lugar, resulta útil para evaluar la significación de los coeficientes de los rezagos de las variables endógenas, lo que tiene una clara interpretación económica en el marco de la teoría de la reacción en cadena. En segundo lugar, este enfoque no requiere conocer a priori el orden de integración de las variables para analizar las relaciones de largo plazo, lo que evita afrontar los problemas asociados a la identificación de raíces unitarias. Así, se eluden también los problemas asociados a la aplicación de los métodos tradicionales de cointegración, que requieren de series largas.

Dado que se incluye en la modelización de cada ecuación la variable dependiente rezagada, la estimación del sistema por mínimos cuadrados ordinarios (MCO) podría presentar problemas de endogeneidad y de correlación con los residuos. Para mitigar estos problemas se utilizan variables instrumentales y se estima el modelo mediante el procedimiento de mínimos cuadrados en tres etapas (MC3E).

Se utilizaron series trimestrales para el período comprendido entre el primer trimestre de 1985 y el cuarto trimestre de 2011 (véase el cuadro A1.1 del anexo). La información utilizada respecto al mercado de trabajo proviene del procesamiento de los microdatos de las Encuestas Continuas de Hogares $(E C H)$ del Instituto Nacional de Estadística (INE) y es representativa de la población urbana del Uruguay (poblaciones de 5.000 y más habitantes) ${ }^{14}$. Sobre la base de las proyecciones de población urbana del INE y del Centro Latinoamericano y Caribeño de Demografía (CELADE)-División de Población de la Comisión Económica para América Latina y el Caribe (CEPAL), se construyó la serie referente a la población urbana en edad de trabajar. La serie correspondiente al salario real proviene de las estadísticas sobre salarios del INE. La serie que corresponde al acervo de capital se construyó llevando a su equivalente trimestral una serie anual disponible en Román y Willebald (2012), considerando una tasa de depreciación constante y utilizando información sobre las inversiones trimestrales de las Cuentas Nacionales del Banco Central del Uruguay (BCU).

Se construyeron dos series de productividad aparente con las que se intenta aproximarse a la evolución de la productividad del capital y del trabajo. La primera se obtuvo a partir del cociente entre el producto interno bruto (PIB) del BCU y el acervo de capital. Esta es una aproximación a la productividad aparente del capital, dado que en el cociente se utiliza el acervo y no el uso del capital, que es el que corresponde. La productividad aparente del trabajo se construyó a partir del cociente entre el PIB y el total de horas trabajadas, dato que se obtuvo del procesamiento de las ECH.

\section{Resultados}

\section{Estimación del sistema de ecuaciones}

La metodología econométrica aplicada requiere, en primer lugar, realizar el contraste de la prueba F a las tres ecuaciones que componen el modelo dinámico multiecuacional: demanda de trabajo, oferta de trabajo y salarios. Dicha prueba contrasta la hipótesis nula $(\mathrm{HO})$ de no existencia de cointegración entre las variables. En los tres casos, el estadístico F se ubicó por encima del valor crítico de las tablas

\footnotetext{
${ }^{14}$ Se utiliza esta información porque recién a partir de 2006 la encuesta cubre también las localidades más pequeñas y las zonas rurales.
} 
de Pesaran, Shin y Smith (2001), por lo que se pudo rechazar HO y concluir que existe una relación de largo plazo entre las variables incluidas en cada modelo (véase el cuadro A1.2 del anexo).

Una vez confirmada la existencia de una relación de largo plazo, se estimó el modelo dinámico multiecuacional. Los resultados que se comentan a continuación son los obtenidos mediante la estimación del sistema de ecuaciones utilizando el método de MC3E (véase el cuadro 1) ${ }^{15}$.

\section{Cuadro 1}

Estimación de las ecuaciones del mercado de trabajo según el método de mínimos cuadrados en tres etapas (MC3E) ${ }^{a}$

\begin{tabular}{|c|c|c|c|c|c|c|c|c|}
\hline \multicolumn{3}{|c|}{ Demanda de trabajo $n_{t}$} & \multicolumn{3}{|c|}{ Oferta de trabajo $I_{t}$} & \multicolumn{3}{|c|}{ Salarios $w_{t}$} \\
\hline Variable & Coeficiente & Valor $p$ & Variable & Coeficiente & Valor $p$ & Variable & Coeficiente & Valor $p$ \\
\hline$n_{t-1}$ & 0,876 & 0,00 & $l_{t-1}$ & 0,774 & 0,00 & $w_{t-1}$ & 0,786 & 0,00 \\
\hline$w_{t-4}$ & $-0,055$ & 0,00 & $u_{t-1}$ & $-0,086$ & 0,02 & $\operatorname{prn}_{t-1}$ & 0,095 & 0,00 \\
\hline$k_{t-1}$ & 0,056 & 0,01 & $\Delta w_{t}$ & 0,093 & 0,01 & $\Delta p r n_{t-1}$ & 0,053 & 0,03 \\
\hline$p r k_{t-1}$ & 0,081 & 0,00 & $z_{t-1}$ & 0,336 & 0,00 & $u_{t-1}$ & $-0,441$ & 0,00 \\
\hline$\Delta p r k_{t}$ & 0,092 & 0,00 & & & & & & \\
\hline$d 1$ & $-0,042$ & 0,00 & $d 3$ & $-0,036$ & 0,00 & $d 1$ & $-0,073$ & 0,00 \\
\hline$d 2$ & $-0,035$ & 0,01 & $d 4$ & 0,016 & 0,06 & $d 6$ & $-0,031$ & 0,00 \\
\hline$d 3$ & $-0,061$ & 0,00 & $d 2$ & $-0,037$ & 0,00 & $d 7$ & $-0,046$ & 0,00 \\
\hline \multirow[t]{3}{*}{ C } & 2,163 & 0,00 & $d 5$ & 0,019 & 0,02 & $d 8$ & 0,041 & 0,01 \\
\hline & & & $C$ & $-1,714$ & 0,00 & $d 9$ & 0,043 & 0,00 \\
\hline & & & & & & $C$ & 0,594 & 0,00 \\
\hline $\begin{array}{l}\text { Número de } \\
\text { observaciones }\end{array}$ & \multicolumn{2}{|c|}{103} & \multicolumn{3}{|c|}{103} & & \multicolumn{2}{|c|}{103} \\
\hline $\mathrm{R}^{2}$ & \multicolumn{2}{|c|}{0,98} & \multicolumn{3}{|c|}{0,98} & & \multicolumn{2}{|c|}{0,97} \\
\hline $\mathrm{SSR}^{\mathrm{b}}$ & \multicolumn{2}{|c|}{0,02} & \multicolumn{3}{|c|}{0,02} & & \multicolumn{2}{|c|}{0,02} \\
\hline \multicolumn{4}{|c|}{ Prueba de normalidad conjunta (valor $p$ ) } & & 0,11 & & & \\
\hline \multicolumn{9}{|c|}{ Prueba de autocorrelación conjunta (valor $p$ ) } \\
\hline & & & \multicolumn{2}{|r|}{1 rezago } & 0,16 & & & \\
\hline & & & \multicolumn{2}{|r|}{2 rezagos } & 0,50 & & & \\
\hline & & & \multicolumn{2}{|r|}{3 rezagos } & 0,64 & & & \\
\hline & & & \multicolumn{2}{|r|}{4 rezagos } & 0,57 & & & \\
\hline
\end{tabular}

Fuente: Elaboración propia.

a Variables instrumentales: $n_{t-1}, l_{t-1}, w_{t-1}, w_{t-4}, w_{t-5}, k_{t-1}, k_{t-2}, p r k_{t-1}, p r k_{t-2}, \Delta p r k_{t}, \Delta p r k_{t-1}, u_{t-1}, u_{t-2}, u_{t-3}, u_{t-4}, \Delta w_{t}, \Delta w_{t-1}, \Delta w_{t-2}, z_{t-1}, z_{t-2}$, $z_{t-3}, p^{\prime} n_{t-1}, \operatorname{prn}_{t-2}, \Delta p r n_{t-1}, \Delta p r n_{t-2}, d 1, d 2, d 3, d 4, d 5, d 6, d 7, d 8, d 9$ у $c$.

b Corresponde a la suma de los errores al cuadrado.

Los coeficientes estimados de las variables incluidas en las tres ecuaciones del sistema resultaron significativos y con los signos esperados; además, los residuos de los mismos son bien comportados (normales, sin autocorrelación ni heterocedasticidad), tanto en la estimación del sistema como en la estimación de cada ecuación por separado (véase el cuadro A1.3 del anexo).

Los resultados indican que la demanda de trabajo depende, en primer lugar, de su propio pasado $\left(n_{t-1}\right)$, lo que confirma la existencia de inercia debido a los costos de ajuste que enfrentan los empleadores a la hora de contratar o despedir trabajadores, por ejemplo, el costo asociado al entrenamiento de los nuevos empleados. La estimación arroja un coeficiente de inercia de la demanda

\footnotetext{
${ }^{15}$ Cabe señalar que los resultados obtenidos por el método de MC3E no presentan diferencias relevantes con respecto a los que arroja la estimación del sistema por MCO. Véase el cuadro A1.5 del anexo en Leites y Porras (2013).
} 
laboral de 0,876, que se ubica en un nivel relativamente elevado de acuerdo a las estimaciones referentes a otros países, antes comentadas, lo que indica que choques sobre la demanda de trabajo generarán efectos en la tasa de desempleo que no desaparecerán rápidamente.

Además, como era esperable, la demanda de trabajo tiene una relación negativa con su propio precio (salario real); pero dicha relación no vincula a los dos elementos en forma simultánea, sino que los cambios en el salario real impactan sobre la demanda de trabajo con un rezago de cuatro trimestres. Según el coeficiente estimado, un incremento del 1\% de los salarios reales reduce la demanda de trabajo en el largo plazo un $0,45 \%[=-0,055 /(1-0,876)]$. Cabe señalar que la variable salario real hace referencia al costo del trabajo dependiente, por lo que es de suponer que si se restringiera el universo de la demanda para ese tipo de trabajadores, la elasticidad estimada sería mayor.

Por su parte, tal como ocurre en los demás países, el acervo de capital en el Uruguay explica la demanda de trabajo, en este caso con un rezago. Efectivamente, un aumento del $1 \%$ del acervo de capital genera un incremento total de la demanda de trabajo del 0,45\% $[=0,056 /(1-0,876)]$. Esta elasticidad se ubica en el entorno de los valores estimados para varios de los países, ya comentados. No solamente el acervo de capital resultó significativo para explicar los cambios en la demanda de trabajo, sino también su productividad, en lo referente al nivel y la variación. La elasticidad de la demanda de trabajo de largo plazo respecto al nivel de la productividad del capital se estimó en 0,65, en tanto que la elasticidad de la demanda de trabajo de largo plazo respecto a la variación de la productividad del capital se estimó en 0,74.

En la ecuación de oferta de trabajo el coeficiente de inercia estimado $(0,774)$ es algo inferior al de la demanda de trabajo, pero se sitúa dentro del rango de los valores estimados para los demás países. Al igual que en el caso de los países en que se incluyó la tasa de desempleo como factor explicativo de la oferta, el signo de la elasticidad indica que en el Uruguay también estaría operando el efecto del trabajador desalentado. Eso se traduce en que ante un aumento de 1 punto porcentual de la tasa de desempleo, la oferta de trabajo se reduce un 0,38\% $[=-0,086 /(1-0,774)]^{16}$, lo que indica que entre las personas que están buscando trabajo en ese contexto predomina la idea de que les resultará difícil encontrarlo y, en consecuencia, una proporción de ellas abandonan la búsqueda y se retiran del mercado. Por otra parte, la oferta laboral reacciona positivamente $(0,41 \%)[=0,093 /(1-0,774)]$ ante un incremento del $1 \%$ del salario real, lo que reflejaría que el efecto de sustitución prevalece sobre el efecto renta, es decir, que ante un aumento del salario, crece la cantidad de personas que prefieren sustituir ocio por trabajo. Finalmente, como es de esperar, la población en edad de trabajar es la variable exógena que más explica la evolución de la oferta de trabajo. Así, un incremento de un 1\% de dicha variable provoca un aumento del $1,49 \%$ en la oferta $[=0,336 /(1-0,774)]$, lo que indica una elasticidad que se ubica en el promedio de los valores estimados para los países desarrollados.

La significación de la variable dependiente rezagada en la ecuación de salarios muestra que también los salarios reales presentan cierta inercia $(0,786)$ asociada al efecto de escalonamiento de salarios. La magnitud de este coeficiente se ubica en el entorno de los valores estimados para la mayoría de los países desarrollados, lo que indica también en este caso que choques sobre los salarios generarán efectos en la tasa de desempleo que no desaparecerán rápidamente. Por su parte, el coeficiente correspondiente a la tasa de desempleo que se incluyó en dicha ecuación podría estar capturando cierta flexibilidad en la respuesta de los salarios reales a las condiciones del mercado de trabajo, en el sentido de que en un contexto de aumento del desempleo, los trabajadores ocupados pierden poder de negociación de los salarios, lo que genera una presión a la baja en términos reales. Es así como un incremento de 1 punto porcentual de la tasa de desempleo provoca una reducción de largo plazo del 2,06\% de los salarios reales. Si bien toma el mismo signo, esta elasticidad se ubica

\footnotetext{
${ }^{16}$ La variable dependiente está en logaritmo, mientras que la variable de desempleo corresponde a una tasa, por lo que el coeficiente estimado multiplicado por 100 se interpreta como la semielasticidad de la oferta de trabajo respecto a la tasa de desempleo.
} 
levemente por encima de los valores estimados para los demás países cuyas ecuaciones de oferta incluyeron esta variable como factor explicativo de los salarios reales (que van entre un 0,5\% y un 1,5\%). A su vez, los salarios también responden a los cambios en los niveles y en la variación de la productividad del trabajo por hora trabajada. La elasticidad de largo plazo de los salarios respecto a los niveles de la productividad del trabajo se estimó en 0,44 (efecto total) y respecto a su variación en 0,25 . Estos valores son inferiores a los hallados en el caso de los Estados Unidos para 2011 en una especificación comparable (que fueron de 1,0 y 3,29, respectivamente).

En las tres ecuaciones hubo que incluir además variables ficticias, en la mayoría de los casos debido a valores puntuales atípicos en las series, o a sucesos de la economía uruguaya que produjeron algún quiebre en las series, como ocurrió con la crisis de 2002 (véase el cuadro A1.1 del anexo).

El sistema estimado permite predecir la evolución de la tasa de desempleo del Uruguay de los últimos 27 años de forma adecuada. Ello se puede observar en el gráfico 2, en que se presenta la evolución real de la tasa de desempleo $\left(l_{t}-n_{t}\right)$, así como la evolución estimada a partir del sistema de ecuaciones (con y sin interacción dinámica) ${ }^{17}$.

Gráfico 2

Evolución de la tasa de desempleo real y estimada a partir del sistema de ecuaciones, 1985-2011

(En porcentajes)

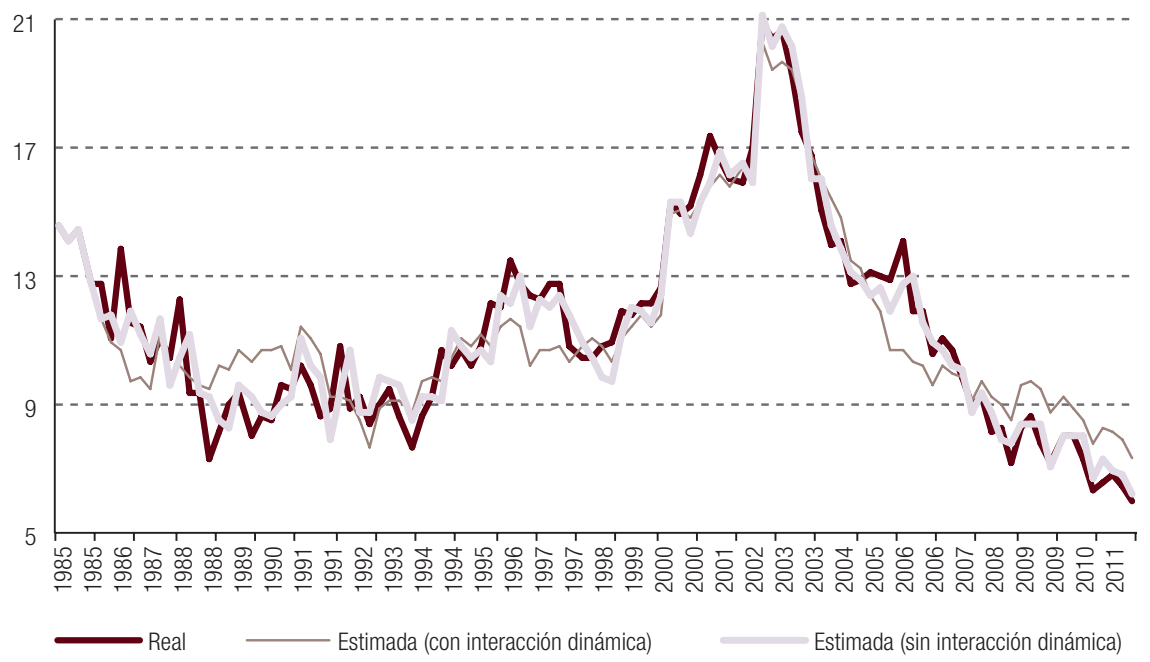

Fuente: Elaboración propia.

\section{Contraste de hipótesis e implicaciones}

Los resultados de las estimaciones permiten dar respuesta a las primeras tres interrogantes planteadas. En el cuadro 2 se presentan las hipótesis y los estadísticos de contraste, y se resumen sus resultados. En primer lugar, en las tres ecuaciones se confirma la significación de las variables dependientes rezagadas $\left(\alpha_{i} \neq 0\right)$, lo que representa evidencia sobre la presencia de inercia en los procesos de ajuste en el mercado de trabajo $(\mathrm{Ha})$. En segundo lugar, se encuentra que el acervo de capital y su productividad inciden de forma significativa en la demanda de trabajo; que la población en edad de trabajar incide en la oferta de trabajo, y que la productividad del trabajo incide en el salario, por lo que se confirma la incidencia de las variables exógenas sobre la tasa de desempleo $\left(\beta_{i} \neq 0, \mathrm{Hb}\right)$.

\footnotetext{
${ }^{17}$ La inclusión de la interacción dinámica implica que la propia tasa de desempleo que estima el sistema es la que se incorpora en las estimaciones sucesivas de la oferta de trabajo y los salarios. La proyección que no incorpora la interacción dinámica toma el valor real de la tasa de desempleo como variable explicativa de la oferta y de los salarios.
} 
Finalmente, el salario es relevante para explicar la demanda y la oferta de trabajo, al mismo tiempo que el desempleo lo es para explicar tanto el salario como la oferta de trabajo, confirmándose así la significación de las interacciones entre las ecuaciones y la presencia de efectos de derrame $\left(\gamma_{i} \neq 0, \mathrm{Hc}\right)$.

Cuadro 2

Contrastes de hipótesis ${ }^{a}$

\begin{tabular}{|c|c|c|c|c|c|}
\hline \multirow{2}{*}{ Hipótesis } & & \multicolumn{3}{|c|}{ Ecuaciones $^{b}$} & \multirow{2}{*}{ Conclusión } \\
\hline & & Demanda & Oferta & Salarios & \\
\hline \multirow{2}{*}{$\begin{array}{l}\text { Ha: presencia de efecto inercia H0: } \\
\text { coeficiente }=0 \mathrm{H} 1 \text { : coeficiente } \neq 0\end{array}$} & Coeficiente estimado & $\alpha_{n}=0,876$ & $\alpha_{l}=0,774$ & $\alpha_{w}=0,786$ & \multirow{2}{*}{$\begin{array}{l}\text { No se rechaza la } \\
\text { presencia de inercia }\end{array}$} \\
\hline & Prueba t (valor $p$ ) & 0,00 & 0,00 & 0,00 & \\
\hline \multirow{6}{*}{$\begin{array}{l}\text { Hb: incidencia de variables exógenas } \\
\text { HO: coeficiente }=0 \mathrm{H} 1 \text { : coeficiente } \neq 0\end{array}$} & Coeficiente estimado & $\beta_{1}=0,056$ & $\beta_{4}=0,336$ & & \multirow{6}{*}{$\begin{array}{l}\text { No se rechaza } \\
\text { la incidencia de } \\
\text { variables exógenas }\end{array}$} \\
\hline & Prueba t (valor $p$ ) & 0,01 & 0,00 & & \\
\hline & Coeficiente estimado & $\beta_{2}=0,081$ & & & \\
\hline & Prueba t (valor $p$ ) & 0,00 & & & \\
\hline & Coeficiente estimado & $\beta_{3}=0,092$ & & & \\
\hline & Prueba t (valor $p$ ) & 0,00 & & & \\
\hline \multirow{4}{*}{$\begin{array}{l}\text { Hc: presencia de efectos de derrame } \\
\text { HO: coeficiente }=0 \mathrm{H} 1 \text { : coeficiente } \neq 0\end{array}$} & Coeficiente estimado & $\gamma_{1}=-0,055$ & $\gamma_{2}=-0,086$ & $\gamma_{4}=-0,441$ & \multirow{4}{*}{$\begin{array}{l}\text { No se rechaza la } \\
\text { existencia de efectos } \\
\text { de derrame }\end{array}$} \\
\hline & Prueba t (valor $p$ ) & 0,00 & 0,02 & 0,00 & \\
\hline & Coeficiente estimado & & $\gamma_{3}=0,093$ & & \\
\hline & Prueba t (valor $p$ ) & & 0,01 & & \\
\hline
\end{tabular}

Fuente: Elaboración propia.

a Corresponden a los contrastes de hipótesis a partir de los resultados de las estimaciones de las ecuaciones del sistema presentadas en el cuadro 1.

b Las ecuaciones son las siguientes:

Demanda: $n_{t}=\alpha_{n} n_{t-1}+\beta_{1} k_{t-1}+\beta_{2} p r k_{t-1}+\beta_{3} \Delta p r k_{t}+\gamma_{1} w_{t-4}$

Oferta: $l_{t}=\alpha_{l} l_{t-1}+\beta_{4} z_{t-1}+\gamma_{2} u_{t-1}+\gamma_{3} \Delta w_{t}$

Salarios: $w_{t}=\alpha_{w} w_{t-1}+\beta_{5} p n_{t-1}+\beta_{6} \Delta p r n_{t}+\gamma_{4} u_{t-1}$

Donde:

$n$ : Número de ocupados; $w$ : Salario real medio; $k$ : Acervo de capital; prk: Productividad aparente del capital; $l$ : Cantidad de personas activas en el mercado de trabajo; $u$ : Tasa de desempleo; $z$ : Población en edad de trabajar, y prn: Productividad aparente del trabajo.

La confirmación de estas tres hipótesis sugiere que una perturbación temporal sobre alguna de las variables del mercado laboral puede generar un efecto persistente en el nivel de desempleo. Para evaluar la magnitud de estos efectos, se realizaron ejercicios de simulación, provocando, en primer lugar, choques sobre la demanda, la oferta y los salarios. Esto permite medir la persistencia temporal de sus efectos en la tasa de desempleo. En segundo lugar, se simularon choques sobre las variables exógenas al mercado laboral y, finalmente, también se evaluó si los procesos de ajuste rezagados son complementarios y amplifican sus efectos (hipótesis Hd).

\section{a) Choques sobre la demanda, la oferta y los salarios}

Se simularon dos tipos de choques sobre las ecuaciones de demanda de trabajo, fijación de salarios y oferta de trabajo: uno puntual o por una sola vez y otro autorregresivo del tipo $A R(1) \operatorname{con} \rho=0,5$. Los efectos de los choques se normalizaron de forma que el impacto inicial sobre la tasa de desempleo fuera de 1 punto porcentual. 
Como se observa en los gráficos 3A y 3B, los choques generan dinámicas diferentes sobre el desempleo según provengan de la demanda, de la oferta o de los salarios. Los choques puntuales en la demanda y en la oferta producen un aumento del desempleo ${ }^{18}$, con efectos que persisten por 11 y 8 trimestres, respectivamente. Los efectos del choque se reducen más de un $90 \%$ en el primer caso recién pasado el noveno trimestre y en el segundo caso pasado el sexto trimestre. Por su parte, el choque en los salarios tiene un efecto inmediato menos relevante, pero pasado un año su incidencia en el desempleo se hace más significativa, dado que el incremento de los salarios impacta negativamente en la demanda de trabajo, con un rezago de cuatro trimestres y los efectos persisten por más de cinco años.

Gráfico 3

Trayectoria del desempleo en respuesta a choques sobre la demanda de trabajo, la oferta de trabajo y los salarios

(En puntos porcentuales)

A. Choque puntual

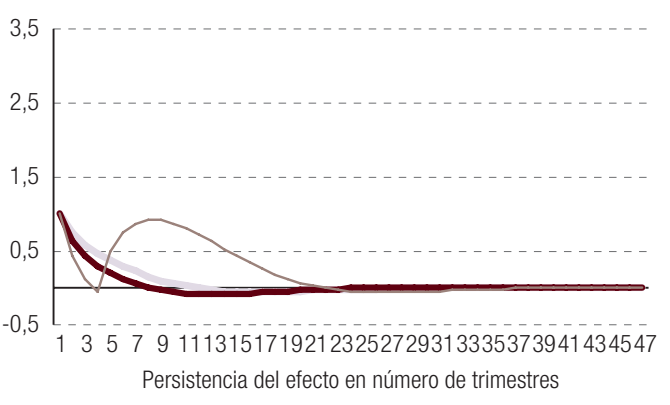

Choque sobre la demanda
B. Choque del tipo $\operatorname{AR}(1)$ con $\rho=0,5$

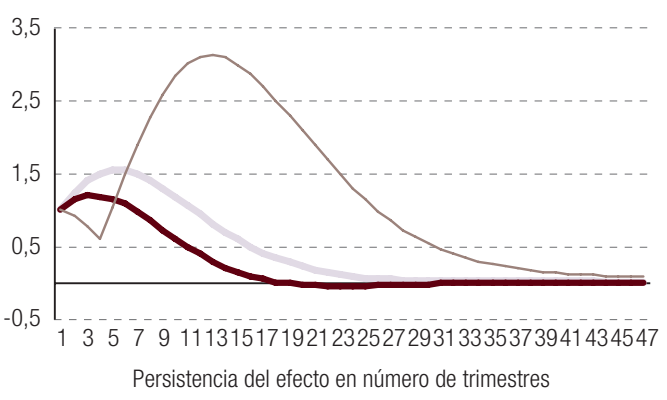

Choque sobre la oferta

— Choque sobre los salarios

Fuente: Elaboración propia.

La respuesta del desempleo a choques del tipo $A R(1)$ muestra un efecto de sobrerreacción y en el largo plazo una alta persistencia (los efectos tardan en desaparecer entre 5 y 13 años, aproximadamente). En relación con los salarios, el desempleo muestra un efecto de sobrerreacción más marcado, que se traduce en un incremento prácticamente constante hasta avanzado el tercer año, y luego efectos que van desapareciendo con mucha lentitud. Cabe mencionar que la mayor persistencia sobre el desempleo de los choques salariales en comparación con los provenientes de la demanda o de la oferta fue constatada también en el estudio referente al Reino Unido (Henry, Karanassou y Snower, 2000).

\section{b) Choques sobre las variables exógenas}

Este ejercicio permite ilustrar cómo la interacción de las variables exógenas junto con los ajustes rezagados genera efectos persistentes en el nivel de desempleo de largo plazo.

Para cada variable exógena se simularon tres tipos de choques: uno puntual (sin memoria) y dos del tipo $A R(1)$, con $\rho=0,5$ y con $\rho=0,9$. En todos los casos se normalizaron los efectos de forma que la incidencia inicial sobre el desempleo correspondiera a una variación de 1 punto porcentual de su tasa. Los resultados se presentan en los gráficos 4A a 4D.

18 En este caso se supone un choque negativo sobre la demanda de trabajo que provoca aumentos del desempleo. 


\section{Gráfico 4}

Efectos en la tasa de desempleo de choques sobre las variables exógenas

(En puntos porcentuales)

A. Acervo de capital

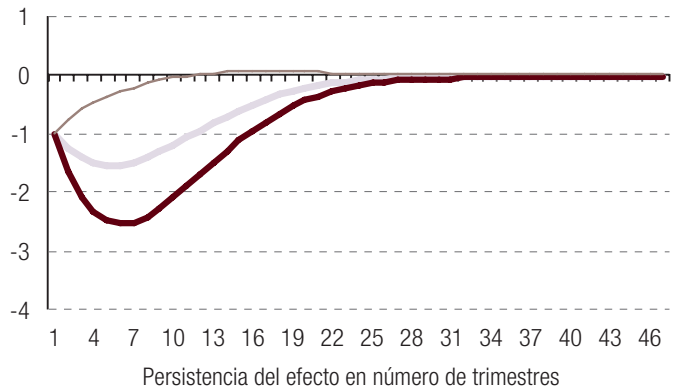

C. Población en edad de trabajar

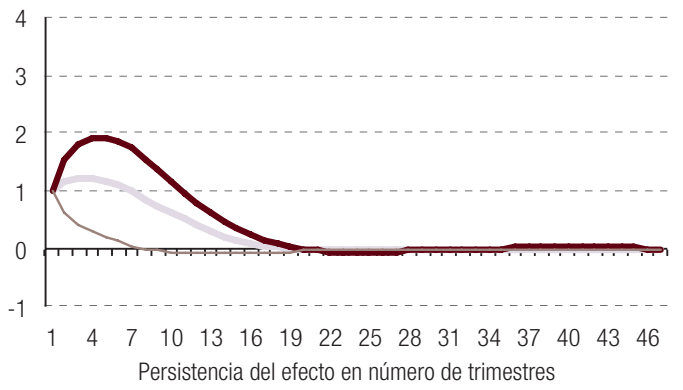

B. Productividad del capital

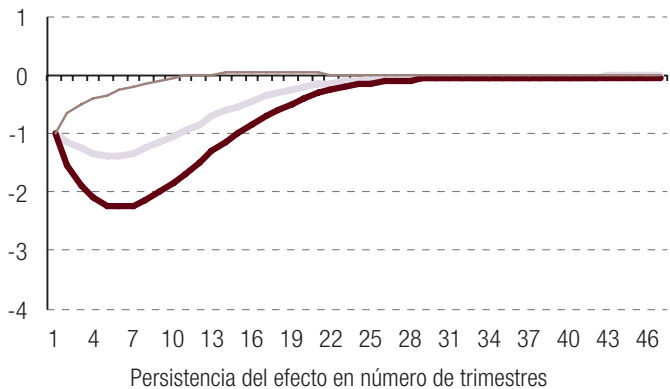

D. Productividad del trabajo

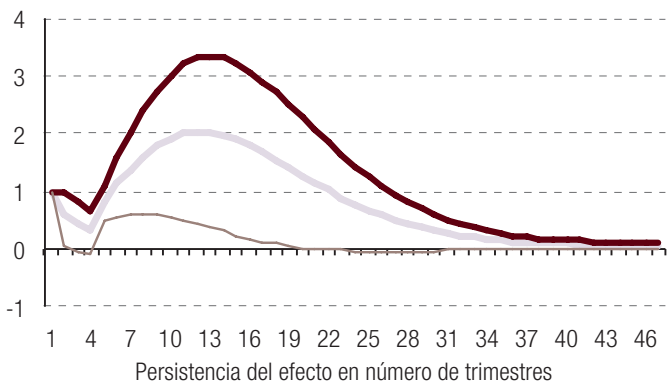

Choque puntual Choque del tipo $\operatorname{AR}(1) \operatorname{con} \rho=0,5 \simeq$ Choque del tipo AR(1) con $\rho=0,9$

Fuente: Elaboración propia.

En el caso de los choques sobre el acervo de capital, la productividad del capital y la población en edad de trabajar, se observa una trayectoria similar del desempleo. Ante una perturbación puntual, el desempleo reacciona y luego vuelve gradualmente a su nivel inicial; en cambio, cuando el choque tiene memoria, se observa en primer lugar una sobrerreacción, que en algunos casos duplica con creces el efecto inicial, y luego un proceso de desaparición muy lento. En lo que se refiere al impacto que tiene un choque proveniente del acervo de capital, se puede observar que sus efectos persisten hasta diez trimestres cuando la perturbación es puntual y hasta 25 trimestres si el choque tiene rezagos.

Los efectos en el desempleo de las perturbaciones sobre la productividad del trabajo no presentan un comportamiento tan gradual como en los casos anteriores. Se observa en primer lugar una caída inmediata del desempleo y un posterior efecto de sobrerreacción debido a la incidencia del salario rezagado en la demanda. El efecto sobre el desempleo alcanza en los períodos subsiguientes una magnitud muy superior a su incidencia inicial, llegando a un máximo de 3,34 puntos porcentuales, y también es más persistente (desaparece recién en un plazo que puede variar entre los 5 y los 17 años, dependiendo del tipo de choque).

\section{c) Complementariedad de los procesos}

Para abordar empíricamente la magnitud de las complementariedades de los procesos de ajuste se sigue a Henry, Karanassou y Snower (2000). Se toma como supuesto un choque sobre la demanda de trabajo y se comparan los resultados de las siguientes dos simulaciones: una que considera que 
todos los efectos operan de forma simultánea y otra que es resultado de sumar las contribuciones individuales de cada ecuación.

Para derivar la contribución individual de la ecuación de demanda al proceso de ajuste del desempleo, se fijan en primer lugar todas las variables endógenas del sistema a su valor corriente, con la excepción del rezago del empleo en la ecuación de demanda. De esta forma, la simulación de la perturbación solo recogerá el efecto en la tasa de desempleo de la inercia de la propia demanda laboral. Seguidamente, la contribución individual de la ecuación de oferta ante dicho choque sobre la demanda se obtiene, en este caso, fijando todas las variables endógenas a su valor corriente, menos la correspondiente al rezago de la oferta. Se procede de igual forma para obtener la contribución individual de la ecuación de salarios.

En el siguiente paso, se suman las respuestas individuales y se normaliza de forma que el efecto inmediato del choque sea de 1 punto porcentual sobre el desempleo. De este modo, se obtiene una serie referida al efecto en el desempleo como respuesta a un choque en la demanda, cuando los procesos de ajuste actúan de forma aislada.

Como se observa en el gráfico 5, la complementariedad de los procesos de ajuste redunda en que una perturbación sobre la demanda tenga un efecto amplificado sobre el desempleo y que, además, este demore más tiempo en desaparecer, lo que representa evidencia favorable a la hipótesis Hd. En el caso de un choque puntual, los efectos recién desaparecen a los 11 trimestres, mientras que si los procesos de ajuste no actuaran de forma complementaria sus efectos desaparecerían en menos de la mitad de ese tiempo. Si, además, la perturbación sigue un proceso del tipo AR(1) con $\rho=0,5$, la complementariedad se traduce no solo en que sus efectos sean más duraderos, sino también en que se amplifiquen, generando incluso un efecto de sobrerreacción.

\section{Gráfico 5}

Complementariedad de los procesos de ajuste rezagados: efectos individuales y conjuntos de un choque sobre la demanda laboral en la tasa de desempleo ${ }^{a}$

(En puntos porcentuales)

A. Choque puntual

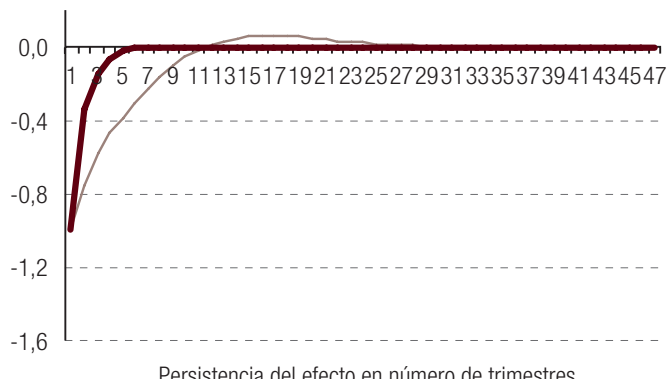

Persistencia del efecto en número de trimestres
B. Choque del tipo $\mathrm{AR}(1) \operatorname{con} \rho=0,5$

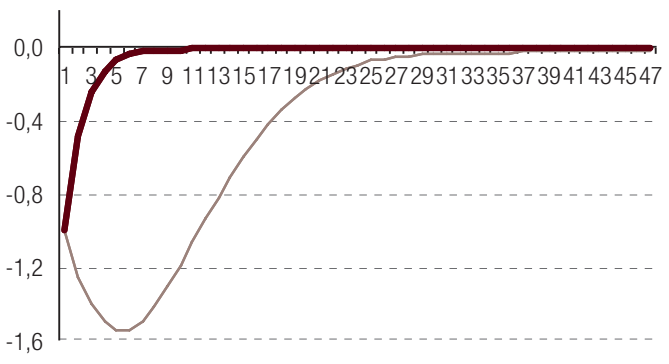

Persistencia del efecto en número de trimestres

$$
\text { — Efecto conjunto } \longrightarrow \text { Suma de los efectos individuales }
$$

Fuente: Elaboración propia.

a Los efectos se calculan mediante dos simulaciones: una que considera que todos los efectos operan de forma simultánea, recogiendo el efecto de las interacciones (efecto conjunto), y otra que es resultado de sumar las contribuciones individuales al proceso de ajuste del desempleo de las ecuaciones de demanda, de oferta y de salarios.

\section{Determinantes de la evolución reciente del desempleo}

Finalmente, este artículo tenía como objetivo explorar qué factores explican la caída que ha registrado la tasa de desempleo del Uruguay, desde los elevados niveles de la crisis de 2002 hasta los guarismos históricamente bajos de los últimos años. Para ello, el análisis se concentró en el período que comienza en el primer trimestre de 2003 y se extiende hasta el cuarto trimestre de 2011. En primer 
lugar, se distinguieron los cambios que evidenció la tasa de desempleo en este período, analizando la contribución de la inercia en los procesos de ajuste. En segundo lugar, se consideró la contribución de la complementariedad de los procesos de ajuste y, finalmente, se profundizó en la incidencia de las variables exógenas en la trayectoria del desempleo de este período.

Siguiendo a Karanassou y Snower (1998), se estimó la contribución de los procesos de inercia a la caída de la tasa de desempleo $\left(\Delta \mathrm{u}_{2011-2003}\right)$. Para ello, en primer lugar, se calculó la variación total de la tasa de desempleo entre 2003 y 2011, utilizando el sistema de ecuaciones completo $\left(\Delta \mathrm{u}_{2011-2003}\right)$. El resultado fue que, en presencia de todos los ajustes, en este período la tasa de desempleo se redujo 10,69 puntos porcentuales. En segundo lugar, se calculó esta misma variación, pero suponiendo que en el sistema no operan los ajustes rezagados de las variables endógenas ( $\left.\Delta u^{n}{ }_{2011-2003}\right)$. Se tomó como supuesto que estas variables se ubican en su nivel corriente, lo que significa que el proceso de ajuste se completa en cada período. Este procedimiento arrojó una caída de la tasa de 5,62 puntos porcentuales y permitió medir cuál hubiera sido la variación del desempleo si solo hubieran operado los cambios en las variables exógenas. De la diferencia entre ambas variaciones se obtiene la contribución de la inercia y de las interacciones, que en este caso corresponde a casi la mitad de la reducción de la tasa de desempleo $\left(\Delta \mathrm{u}_{2011-2003}=\Delta \mathrm{u}_{2011-2003}-\Delta \mathrm{u}_{2011-2003}=5,07\right.$ puntos porcentuales) (véase el cuadro 3), mientras que el resto corresponde a los efectos de las variables exógenas.

Cuadro 3

Contribución de los ajustes rezagados a la variación de la tasa de desempleo entre 2003 y 2011

(En puntos porcentuales)

\begin{tabular}{|c|c|c|c|c|}
\hline & & & & $\begin{array}{l}\text { Variación de la tasa } \\
\text { de desempleo }\end{array}$ \\
\hline Total & & $\Delta \mathrm{u}_{2011-2003}$ & $=$ & $-10,69$ \\
\hline Sin ajustes rezagados en variables endógenas & & $\Delta \mathrm{u}_{2011-2003}$ & $=$ & $-5,62$ \\
\hline Contribución conjunta de los ajustes rezagados & & $\Delta \mathrm{u}_{2011-2003}^{\wedge}$ & $=$ & $-5,07$ \\
\hline Solo con inercia en la demanda & & $\Delta \mathrm{u}(\mathrm{EA})_{2011-2003}$ & $=$ & $-6,25$ \\
\hline Solo con inercia en los salarios & & $\Delta u(W F)_{2011-2003}$ & $=$ & $-5,61$ \\
\hline Solo con inercia en la oferta & & $\Delta \mathrm{u}(\mathrm{LF})_{2011-2003}$ & $=$ & $-4,93$ \\
\hline \multicolumn{5}{|l|}{ Contribuciones individuales de la inercia } \\
\hline & $\Delta \mathrm{u}^{\wedge}(\mathrm{EA})_{2011-2003}=\Delta \mathrm{u}(\mathrm{EA})_{2011-2003}$ & $-\Delta u_{2011-2003}^{n}$ & $=$ & $-0,63$ \\
\hline & $\Delta u^{\wedge}(W F)_{2011-2003}=\Delta u(W F)_{2011-2003}$ & $-\Delta u_{2011-2003}^{n}$ & $=$ & 0,01 \\
\hline & $\Delta \mathrm{u}^{\wedge}\left(\mathrm{LF}_{2011-2003}=\Delta \mathrm{u}\left(\mathrm{LF}_{2011-2003}\right.\right.$ & $-\Delta u_{2011-2003}^{n}$ & $=$ & 0,69 \\
\hline
\end{tabular}

Contribución conjunta de los efectos individuales

$$
\Delta u^{\wedge}(E A)_{2011-2003}+\Delta u^{\wedge}(W F)_{2011-2003}+\Delta u^{\wedge}(L F)_{2011-2003}=\quad 0,07
$$

Fuente: Elaboración propia.

Nota: $\quad \Delta \mathrm{u}$ : Variación del desempleo proyectado; $\Delta \mathrm{u}^{n}$ : Variación del desempleo sin inercia; $\Delta \mathrm{u}^{\wedge}$ : Variación del desempleo debido a inercia; EA: Inercia en la demanda; LF: Inercia en la oferta; WF: Inercia en los salarios.

Para medir la relevancia de la complementariedad de los efectos de ajuste rezagado se propone simular cuál hubiera sido la variación de la tasa de desempleo entre estos años suponiendo que dicha complementariedad no existe. Para estimar la variación sin complementariedad de los ajustes, se suman las contribuciones de la inercia individual de cada variable. Por ejemplo, en el caso de la demanda de trabajo $\left(\Delta u^{\wedge}(E A)_{2011-2003}\right)$, la contribución individual de la inercia surge de la diferencia entre la variación de la tasa de desempleo suponiendo solo inercia en la demanda $\left(\Delta u(E A)_{2011-2003}\right)$ y la variación de la tasa de desempleo sin ningún proceso de inercia $\left(\Delta u^{n} 2011-2003\right)$. De la misma forma, se estima la contribución de la inercia de la oferta laboral $\left(\Delta u^{\wedge}(L F)_{2011-2003}\right)$ y de los salarios $\left(\Delta u^{\wedge}(W F)_{2011-2003}\right)$. Como se observa en el cuadro 3, la tasa de desempleo hubiera registrado 
un aumento de 0,07 puntos porcentuales, en vez de la caída antes mencionada de 5,07 puntos porcentuales. Por otra parte, se puede observar que la contribución a la caída del desempleo entre 2003 y 2011 atribuible a la inercia de la demanda fue de -0,63 puntos porcentuales y la de la oferta laboral de 0,69 puntos porcentuales, mientras que el aporte de los salarios fue casi nulo.

Finalmente, ¿qué hubiera pasado con la tasa de desempleo si las variables exógenas no hubieran registrado variaciones entre 2003 y 2011 ? Tanto el acervo de capital y su productividad como la población en edad de trabajar y la productividad del trabajo evolucionaron al alza entre esos años. El ejercicio consiste en simular la trayectoria del desempleo alterando el valor de las variables exógenas.

Como punto de referencia se considera la serie correspondiente a la tasa de desempleo que surge de la estimación del sistema de ecuaciones completo. Seguidamente, para calcular la contribución del acervo de capital a la evolución de la tasa de desempleo, se simuló la trayectoria del desempleo tomando como supuesto que el acervo de capital se mantuvo cada trimestre del período comprendido entre 2004 y 2011 en el mismo nivel de igual trimestre de 2003, considerando en la estimación los valores reales de las demás variables exógenas. La contribución de dicha variable se obtiene como resultado de la diferencia entre ambas series. Este procedimiento se aplica para todas las variables exógenas.

En el gráfico 6 se presentan los resultados. Cuando las diferencias toman valores positivos, significa que si la variable exógena hubiera mantenido los valores de 2003 y no hubiera evolucionado como lo hizo, la tasa de desempleo hubiera sido más alta. De acuerdo a este ejercicio, los incrementos de la productividad del capital y de la acumulación de capital parecen haber hecho una contribución relevante para explicar la trayectoria reciente del desempleo. Esto significa que si el acervo de capital y su productividad no hubieran evolucionado al alza, sino que se hubieran manteniendo en los niveles que registraban en 2003, la tasa de desempleo habría registrado una caída menor y se hubiera ubicado 3,7 puntos porcentuales y 7,5 puntos porcentuales, respectivamente, por encima del nivel efectivamente alcanzado ${ }^{19}$.

\section{Gráfico 6}

Contribución de las variables exógenas a la caída de la tasa de desempleo, 2003-2011

(En puntos porcentuales)

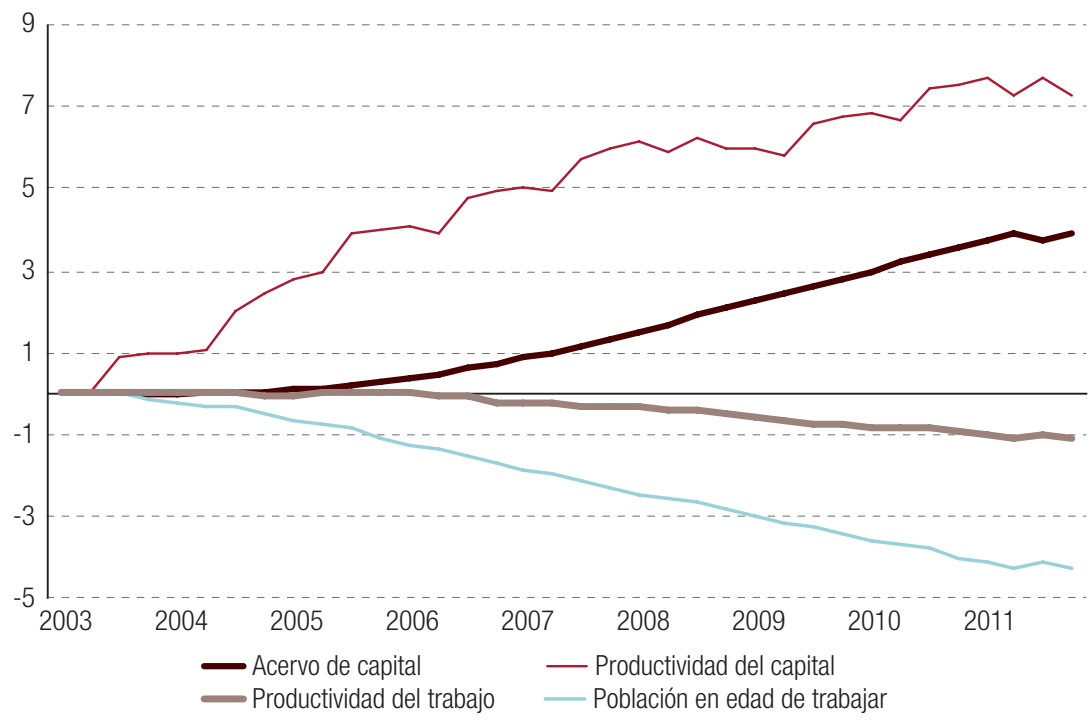

Fuente: Elaboración propia. ${ }^{19}$ El nivel efectivamente alcanzado no es el efectivamente observado, sino el que surge de la estimación del sistema en su
conjunto. 
Las demás variables operaron en sentido inverso. El aumento de la población en edad de trabajar origina un incremento de la oferta laboral, presionando al alza el desempleo. Si esta variable hubiera mantenido su nivel de 2003, la tasa de desempleo en 2011 hubiera sido 4 puntos porcentuales inferior. La productividad del trabajo, por su parte, fue una variable que aumentó en dicho período y presionó directamente al alza los salarios, al mismo tiempo que influyó indirectamente en cambios en la demanda y en la oferta, con lo que también tuvo un impacto en el sentido de aumentar el desempleo, aunque muy inferior al de la población en edad de trabajar.

A modo de síntesis, puede afirmarse que en el gráfico 6 se ilustran evoluciones consistentes con lo esperado. Muestra que el crecimiento registrado en el acervo de capital y en su productividad, junto con la complementariedad de los ajustes del mercado de trabajo del Uruguay, permitirían explicar gran parte de la significativa caída de la tasa de desempleo que se produjo entre 2003 y 2011.

\section{Conclusiones}

Se aplicó el enfoque de la teoría de la reacción en cadena para estudiar la dinámica del desempleo en el Uruguay durante el período comprendido entre 1985 y 2011, aportando evidencia que contribuye a entender los determinantes del nivel de desempleo y a explicar la caída que se produjo en la tasa de desempleo desde 2003 en adelante.

Por un lado, se constató que las variables relevantes del mercado de trabajo, demanda, oferta y formación de salarios, presentan inercia y que, además, por el hecho de estar interconectadas, generan efectos de derrame. Ello se traduce en que choques puntuales sobre alguna de las variables del mercado de trabajo tengan efectos sobre el desempleo que demoran en desaparecer. Si, por ejemplo, la perturbación proviene de la demanda o de la oferta, se generan efectos sobre el desempleo que persisten por 11 trimestres y 8 trimestres, respectivamente, mientras que si el choque proviene del salario, los efectos se prolongan hasta por cinco años.

Por otro lado, los procesos de ajuste operan de forma complementaria, ocasionando efectos en los que se propaga la magnitud y persistencia de los choques. Si no existiera dicha complementariedad, un choque puntual que incrementara la demanda de trabajo provocando una caída de 1 punto porcentual en la tasa de desempleo se desvanecería al quinto trimestre, mientras que, como se indicó anteriormente, el efecto perdura durante 11 trimestres.

Además, uno de los aportes esenciales del enfoque de la teoría de la reacción en cadena es que permite detectar la influencia de las variaciones de variables exógenas al mercado de trabajo sobre la tasa de desempleo. En particular, se pudo observar que las variaciones del acervo de capital generan efectos persistentes sobre el desempleo, que demoran en desaparecer entre tres y siete años, dependiendo del tipo de choque.

En relación con el desempeño reciente del desempleo en el Uruguay, se encontró que la reducción de los niveles de desempleo estaría explicada fundamentalmente por dos factores. En primer lugar, entre las variables exógenas, se destaca por su importancia el aumento de la productividad del capital y del acervo de capital. Si estas variables hubieran mantenido los niveles que presentaban en 2003, la tasa de desempleo habría registrado una caída menor y se habría ubicado 3,7 puntos porcentuales y 7,5 puntos porcentuales, respectivamente, por encima del nivel efectivamente alcanzado. En segundo lugar, una alta proporción de la caída se explica por los efectos de derrame que surgen de la complementariedad de los procesos de ajuste de las variables del mercado de trabajo uruguayo.

Finalmente, los resultados de este trabajo tienen algunas implicaciones relevantes, tanto en relación con la dinámica del desempleo como con el diseño de futuras políticas. En primer lugar, el haber constatado la presencia de inercia y complementariedad en los procesos de ajuste de 
las variables del mercado de trabajo, así como la existencia de efectos de derrame con impactos persistentes, sugiere que la tasa de desempleo no converge a un nivel invariante. Por el contrario, su evolución de largo plazo estaría determinada por la secuencia infinita de choques nominales y reales, y mecanismos intertemporales de propagación. Por lo tanto, pierde peso el argumento que sugiere que los niveles actuales de empleo (y de bajo desempleo) no son sostenibles en el tiempo. Por otra parte, Karanassou, Sala y Salvador (2008) argumentan que ante perturbaciones de diferente duración se pueden requerir políticas distintas y sostienen que la teoría de la reacción en cadena abre el espacio para la aplicación de políticas de combate al desempleo. Por ejemplo, intervenciones dirigidas a promover incrementos en el acervo de capital y/o en la productividad de los factores podrían tener efectos persistentes sobre la tasa de desempleo de corto y largo plazo.

\section{Bibliografía}

Badagián, A. y otros (2001), "Tasa de desempleo de Montevideo: ¿raíz unitaria o cambio estructural?", serie Documentos de Trabajo, № DT (01/01), Montevideo, Universidad de la República.

Banco Mundial (2012), El papel del mercado laboral en la transformación de América Latina, Washington, D.C.

Bande, R. (2002), "Ajustes dinámicos en las tasas de paro: España vs. Portugal", Análise Económica, Nㅜ 20, Instituto Universitario de Estudios y Desarrollo de Galicia.

Bande, R. y M. Karanassou (2009), "Labour market flexibility and regional unemployment rate dynamics: Spain 1980-1995", Papers in Regional Science, vol. 88, № 1, Wiley Blackwell.

Borraz, F. y M. Tubio (2009), "La tasa natural de desempleo en Uruguay", Banco Central del Uruguay [en línea] www.bcu.gub.uy/autoriza/peiees/jor/2009/iees03j3601009.pdf.

González, I. y H. Sala (2011), "Macroeconomic consequences of the U.S. financialisation process: lower capital accumulation, higher unemployment" [en línea] http://www.ecap.uab.es/secretaria/trebrecerca/ Igonzalez.pdf.

Henry, B., M. Karanassou y D. Snower (2000), "Adjustment dynamics and the natural rate: an account of UK unemployment”, Oxford Economic Papers, vol. 52, № 1, Oxford University Press.

Karanassou, M. (1998), "Unemployment dynamics: the chain reaction theory of unemployment", tesis, Londres, Birkbeck College, Universidad de Londres.

Karanassou, M. y D. Snower (2004), "Unemployment invariance", German Economic Review, vol. 5, № 3, Wiley. (2000), "Characteristics of unemployment dynamics: the chain reaction approach", IZA Discussion Paper, $N^{\circ} 127$, Bonn, Institute for the Study of Labour (IZA).

_ (1998), "How labour market flexibility affects unemployment: Iong term implications of the chain reaction theory", The Economic Journal, vol. 108, № 448, Wiley.

(1997), "Is the natural rate a reference point?", European Economic Review, vol. 41, № 3-5, Amsterdam, Elsevier.

(1996), "Explaining disparities in unemployment dynamics", The 1990's Slump: Causes and Cures, M. Baldassari, L. Paganetto y E. Phelps (eds.), Londres, Palgrave Macmillan.

Karanassou, M. y H. Sala (2010), "Labour market dynamics in Australia: what drives unemployment?", Economic Record, vol. 86, № 273, The Economic Society of Australia.

(2008), "The rise and fall of Spanish unemployment: a chain reaction theory perspective", Working Paper, $^{\circ} 273$, Queen Mary University of London.

Karanassou, M., H. Sala y F. Salvador (2008), "The (ir)relevance of the NRU for policy making: the case of Denmark", Scottish Journal of Political Economy, vol. 55, № 3, Scottish Economic Society.

Karanassou, M., H. Sala y D. Snower (2009), "Phillips curves and unemployment dynamics: a critique and a holistic perspective", Journal of Economic Surveys, vol. 23, $N^{\circ} 4$.

(2003), "Unemployment in the European Union: a dynamic reappraisal", Economic Modelling, vol. 20, $^{\circ} 2$, Amsterdam, Elsevier.

Layard, R., S. Nickell y R. Jackman (1991), Unemployment: Macroeconomic Performance and the Labour Market, Oxford, Oxford University Press.

Leites, M. y S. Porras (2013), "El enfoque de la reacción en cadena: una aplicación para explicar la dinámica del desempleo en Uruguay", serie Documentos de Trabajo, N DT 11/2013, Montevideo, Instituto de Economía, Universidad de la República. 
Montuenga, V. y V. Ramos (2005), "Reconciling the wage curve and the Phillips curve", Journal of Economic Surveys, vol. 19, № 5 , Wiley.

Pesaran, H. (1997), "The role of economic theory in modelling the long run", The Economic Journal, vol. 107, $N^{\circ} 440$, Wiley.

Pesaran, H. y Y. Shin (1995), "An autoregresive distributed lag modelling approach to cointegration analysis", DEA Working Paper, № 9514.

Pesaran, H., Y. Shin y R. Smith (2001), "Bounds testing approaches to the analysis of level relationships", Journal of Applied Econometrics, vol. 16, № 3, Wiley. (1996), "Testing for the existence of a long run relationship", CREST Working Paper, № 9645.

Phelps, E.S. (1994), Structural Slumps, Cambridge, Massachusetts, Harvard University Press.

Rodríguez, S. (1998), "Modelización y desestacionalización de la tasa de desempleo de Montevideo", documento presentando en las 13 Jornadas Anuales de Economía del Banco Central del Uruguay.

Román, C. y H. Willebald (2012), "Indicadores de inversión en el largo plazo. Una propuesta para Uruguay (1870-2011)", Documento de Trabajo, № 12-21, Montevideo, Instituto de Economía, Universidad de la República.

Spremolla, A. (2001), "Persistencia en el desempleo de Uruguay", Cuadernos de Economía, vol. 32, № 113, Santiago, Instituto de Economía, Pontificia Universidad Católica de Chile.

Taylor, J. (1979), "Staggered wage setting in a macro model", American Economic Review, vol. 69, № 2, Nashville, Tennessee, American Economic Association.

Weller, J. (2014), "Aspectos de la evolución reciente de los mercados laborales de América Latina y el Caribe", Revista CEPAL, № 114 (LC/G.2629-P), Santiago, Comisión Económica para América Latina y el Caribe (CEPAL). 


\section{Anexo A1}

Cuadro A1.1

Descripción de las variables utilizadas

\begin{tabular}{|c|c|c|c|}
\hline & & & Fuente \\
\hline \multicolumn{4}{|c|}{ Variables endógenas } \\
\hline$l_{t}$ & \multicolumn{2}{|c|}{ Población activa en logaritmo ${ }^{\mathrm{a}}$} & INE \\
\hline$\underline{n_{t}}$ & \multicolumn{2}{|c|}{ Número de ocupados en logaritmo ${ }^{\mathrm{a}}$} & INE \\
\hline$u_{t}$ & \multicolumn{2}{|l|}{ Tasa de desempleo } & $\left(l_{t}-n_{t}\right)$ \\
\hline$\underline{w_{t}}$ & \multicolumn{2}{|l|}{ Salario real en logaritmo } & INE \\
\hline \multicolumn{4}{|c|}{ Variables exógenas y de control } \\
\hline$k_{t}$ & \multicolumn{2}{|c|}{ Acervo de capital en logaritmo ${ }^{b}$} & IECON \\
\hline $\operatorname{prk}_{t}$ & \multicolumn{2}{|c|}{ Productividad del capital en logaritmoc } & BCU/IECON \\
\hline$z_{t}$ & \multicolumn{2}{|c|}{ Población en edad de trabajar en logaritmo ${ }^{d}$} & INE \\
\hline $\mathrm{prn}_{t}$ & \multicolumn{2}{|c|}{ Productividad del trabajo en logaritmo ${ }^{\mathrm{e}}$} & $\mathrm{BCU} / \mathrm{INE}$ \\
\hline \multicolumn{4}{|c|}{ Variables ficticias } \\
\hline$d 1$ & 2002, tercer trimestre & \multicolumn{2}{|l|}{$=1$ crisis económica } \\
\hline$d 2$ & 1997, primer trimestre & \multicolumn{2}{|l|}{$=1$ valor atípico en serie $n_{t}$ y $l_{t}$} \\
\hline$d 3$ & 2000, segundo trimestre & \multicolumn{2}{|l|}{$=1$ valor atípico en serie $n_{t}$ y $l_{t}$} \\
\hline$d 4$ & 1994, cuarto trimestre & \multicolumn{2}{|l|}{$=1$ valor atípico en serie $l_{t}$} \\
\hline$d 5$ & 1987, tercer trimestre & \multicolumn{2}{|l|}{$=1$ valor atípico en serie $l_{t}$} \\
\hline$d 6$ & $\geq 2002$, tercer trimestre & \multicolumn{2}{|l|}{ = 1 salida de la crisis y recuperación económica } \\
\hline$d 7$ & \multicolumn{3}{|c|}{ 1990, primer a cuarto trimestre = 1 alta inflación, caída del salario real } \\
\hline$d 8$ & 1993, primer trimestre & \multicolumn{2}{|l|}{$=1$ valor atípico en serie $w_{t}$} \\
\hline$d 9$ & 1987, cuarto trimestre & \multicolumn{2}{|l|}{$=1$ valor atípico en serie $w_{t}$} \\
\hline
\end{tabular}

Fuente: Instituto Nacional de Estadística (INE), Banco Central del Uruguay (BCU) e Instituto de Economía (IECON) de la Universidad de la República, Uruguay.

a Las series se construyeron a partir de las tasas de actividad y de empleo de las Encuestas Continuas de Hogares (ECH) y de las proyecciones de población del INE.

b Véase una descripción de la elaboración de esta serie anual sobre el acervo de capital en Román y Willebald (2012). Los datos se llevaron a su equivalente trimestral considerando una tasa de depreciación constante y utilizando información sobre las inversiones trimestrales.

c Es resultado del cociente entre el producto interno bruto (PIB) en términos reales y el acervo de capital.

d Proyecciones de población del INE por edades simples.

e Es resultado del cociente entre el PIB y el total de horas trabajadas. 
Cuadro A1.2

Prueba F sobre la relación de largo plazo entre las variables

Demanda de trabajo

\begin{tabular}{|c|c|c|c|c|c|c|}
\hline \multicolumn{7}{|c|}{ Demanda de trabajo } \\
\hline \multicolumn{7}{|c|}{$\Delta n_{t}=a_{1} n_{t-1}+a_{2} w_{t-4}+a_{3} k_{t-1}+a_{4} p r k_{t-1}+\sum a_{n i} \Delta n_{t-i}+\sum a_{w 4}-i \Delta w_{t-4-i}+\sum a_{k i} \Delta k_{t-i}+\sum a_{p r k i} \Delta p r k_{t-i}+a_{0}$} \\
\hline Rezagos & & 1 & 2 & 3 & 4 & 5 \\
\hline \multirow{2}{*}{ Criterios de selección } & Akaike & $-5,55^{\star}$ & $-5,49$ & $-5,45$ & $-5,41$ & $-5,41$ \\
\hline & Schwarz & $-5,27^{\star}$ & $-5,09$ & $-4,96$ & $-4,81$ & $-4,70$ \\
\hline \multirow{2}{*}{ Pruebas de autocorrelación ${ }^{\mathrm{a}}$} & $\underline{S C}\left[\chi^{2}(1)\right]$ & 0,51 & 0,83 & 0,75 & 0,64 & 0,17 \\
\hline & $\operatorname{SC}\left[\chi^{2}(4)\right]$ & 0,83 & 0,86 & 0,65 & 0,40 & 0,19 \\
\hline \multirow{2}{*}{ Prueba $F\left(H_{0}: a_{1}=a_{2}=a_{3}=a_{4}=0\right)^{b}$} & Estadístico & 5,40 & 3,73 & 2,96 & 2,42 & 2,63 \\
\hline & Significación & ** & & & & \\
\hline \multirow{2}{*}{ Prueba t $\left(\mathrm{HO}: \mathrm{a}_{1}=0\right)^{\mathrm{c}}$} & Estadístico & $-3,52$ & $-2,98$ & $-3,13$ & $-2,96$ & $-2,72$ \\
\hline & Significación & * & & & & \\
\hline \multicolumn{7}{|c|}{ Oferta de trabajo } \\
\hline \multicolumn{7}{|c|}{$\Delta l_{t}=b_{1} l_{t-1}+b_{2} w_{t-1}+b_{3} u_{t-1}+b_{4} z_{t-1}+\sum b_{l i} \Delta l_{t-i}+\sum b_{w i} \Delta w_{t-i}+\sum b_{u i} \Delta u_{t-i}+\sum b_{z i} \Delta z_{t-i}+b_{0}$} \\
\hline Rezagos & & 1 & 2 & 3 & 4 & 5 \\
\hline \multirow{2}{*}{ Criterios de selección } & Akaike & $-5,83^{*}$ & $-5,79$ & $-5,73$ & $-5,67$ & $-5,63$ \\
\hline & Schwarz & $-5,52^{*}$ & $-5,38$ & $-5,22$ & $-5,05$ & $-4,91$ \\
\hline \multirow{2}{*}{ Pruebas de autocorrelación ${ }^{a}$} & $\underline{\operatorname{SC}[\chi 2(1)]}$ & 0,97 & 0,64 & 0,39 & 0,54 & 0,94 \\
\hline & $S C\left[\chi^{2}(4)\right]$ & 0,56 & 0,60 & 0,30 & 0,46 & 0,59 \\
\hline \multirow{2}{*}{ Prueba F $\left(\mathrm{HO}: \mathrm{b}_{1}=\mathrm{b}_{2}=\mathrm{b}_{3}=\mathrm{b}_{4}=0\right)^{\mathrm{b}}$} & Estadístico & 5,25 & 3,55 & 3,20 & 2,33 & 2,57 \\
\hline & Significación & ** & & & & \\
\hline \multirow{2}{*}{ Prueba t $\left(H O: b_{1}=0\right)^{c}$} & Estadístico & $-4,40$ & $-3,41$ & $-2,92$ & $-2,28$ & $-2,34$ \\
\hline & Significación & $\star \star \star *$ & & & & \\
\hline \multicolumn{7}{|c|}{ Salarios } \\
\hline \multicolumn{7}{|c|}{$\Delta w_{t}=c_{1} w_{t-1}+c_{2} p r n_{t-1}+c_{3} u_{t-1}+\sum c_{w i} \Delta w_{t-i}+\sum c_{p r n i} \Delta p r n_{t-i}+\sum c_{u i} \Delta u_{t-i}+c_{0}$} \\
\hline Rezagos & & 1 & 2 & 3 & 4 & 5 \\
\hline \multirow{2}{*}{ Criterios de selección } & Akaike & $-5,38$ & $-5,41^{*}$ & $-5,40$ & $-5,37$ & $-5,38$ \\
\hline & Schwarz & $-5,10^{*}$ & $-5,05$ & $-4,97$ & $-4,86$ & $-4,79$ \\
\hline \multirow{2}{*}{ Pruebas de autocorrelación ${ }^{\mathrm{a}}$} & $\underline{S C}\left[\chi^{2}(1)\right]$ & 0,79 & 0,61 & 0,51 & 0,74 & 0,39 \\
\hline & $\operatorname{SC}\left[\chi^{2}(4)\right]$ & 0,37 & 0,73 & 0,75 & 0,63 & 0,38 \\
\hline \multirow{2}{*}{ Prueba F $\left(\mathrm{HO}: \mathrm{C}_{1}=\mathrm{C}_{2}=\mathrm{C}_{3}=0\right)^{\mathrm{d}}$} & Estadístico & 19,48 & 13,31 & 11,35 & 10,94 & 12,82 \\
\hline & Significación & $* * \star$ & $* * *$ & $* \star *$ & $* \star \star$ & *** \\
\hline \multirow{2}{*}{ Prueba t $\left(\mathrm{HO}: \mathrm{C}_{1}=0\right)^{\mathrm{e}}$} & Estadístico & $-6,62$ & $-5,73$ & $-4,89$ & $-4,65$ & $-5,29$ \\
\hline & Significación & 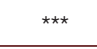 & 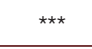 & 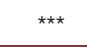 & 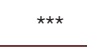 & $\star \star \star *$ \\
\hline
\end{tabular}

Fuente: Elaboración propia.

a Valor $p$.

b Valores críticos al 90\% (*): 2,72-3,77; al 95\% (**): 3,23-4,35; al 99\% (**): 4,29-5,61.

c Valores críticos al $90 \%\left(^{\star}\right):(-2,57)-(-3,46)$; al $95 \%\left(^{* \star}\right):(-2,86)-(-3,78)$; al $99 \%\left(^{\star \star \star}\right):(-3,43)-(-4,37)$.

d Valores críticos al $90 \%\left(^{*}\right): 3,17-4,14$; al $95 \%\left(^{* *}\right): 3,79-4,85$; al $99 \%\left(^{* \star *}\right): 5,15-6,36$.

e Valores críticos al $90 \%\left(^{*}\right):(-2,57)-(-3,21)$; al $95 \%\left(^{* \star}\right):(-2,86)-(-3,53)$; al $99 \%\left(^{* \star *}\right):(-3,43)-(-4,10)$. 
Cuadro A1.3

Pruebas de especificación de las ecuaciones del sistema ${ }^{a}$

\begin{tabular}{|c|c|c|c|c|c|}
\hline \multicolumn{6}{|c|}{ Estimaciones con variables instrumentales $(\mathrm{Vl})^{\mathrm{b}}$} \\
\hline \multicolumn{2}{|c|}{ Demanda de trabajo } & \multicolumn{2}{|c|}{ Oferta de trabajo } & \multicolumn{2}{|c|}{ Salarios } \\
\hline $\operatorname{NOR}\left[\chi^{2}(2)\right]$ & 0,925 & $\operatorname{NOR}\left[\chi^{2}(2)\right]$ & 0,845 & $\operatorname{NOR}\left[\chi^{2}(2)\right]$ & 0,408 \\
\hline $\operatorname{SC}\left[\chi^{2}(1)\right]$ & 0,854 & $\operatorname{SC}\left[\chi^{2}(1)\right]$ & 0,087 & $\operatorname{SC}\left[\chi^{2}(1)\right]$ & 0,538 \\
\hline $\mathrm{SC}\left[\chi^{2}(4)\right]$ & 0,788 & $\operatorname{SC}\left[\chi^{2}(4)\right]$ & 0,043 & $\operatorname{SC}\left[\chi^{2}(4)\right]$ & 0,543 \\
\hline HET $\left[\chi^{2}(6)\right]$ & 0,295 & HET $\left[\chi^{2}(8)\right]$ & 0,148 & $\mathrm{HET}\left[\chi^{2}(7)\right]$ & 0,120 \\
\hline
\end{tabular}

Fuente: Elaboración propia.

a Los valores que se presentan corresponden a los valores $p$ de las pruebas de normalidad (NOR), correlación serial con uno y cuatro rezagos (SC) y heterocedasticidad (HET).

b Variables instrumentales: $n_{t-1}, l_{t-1}, w_{t-1}, w_{t-4}, w_{t-5}, k_{t-1}, k_{t-2}, p r k_{t-1}, p r k_{t-2}, \Delta p r k_{t}, \Delta p r k_{t-1}, u_{t-1}, u_{t-2}, u_{t-3}, u_{t-4}, \Delta w_{t}, \Delta w_{t-1}, \Delta w_{t-2}$, $z_{t-1}, z_{t-2}, z_{t-3}, \operatorname{prn}_{t-1}, \operatorname{prn}_{t-2}, \Delta p r n_{t-1}, \Delta p r n_{t-2}, d 1, d 2, d 3, d 4, d 5, d 6, d 7, d 8, d 9$ у $c$. 